\title{
A Kinetic Beam Scheme for Relativistic Gas Dynamics
}

\author{
Jaw-Yen Yang,*,1 Min-Hung Chen,* I-Nan Tsai, $\dagger$ and Jer-Wei Chang* \\ *Institute of Applied Mechanics, $\dagger$ Department of Mathematics, National Taiwan University, Taipei, 10764 Taiwan, Republic of China
}

Received October 10, 1996; revised March 24, 1997

\begin{abstract}
An extension of the beam scheme of Sanders and Prendergast (Astrophys. J. 188, 489, 1974) for the Newtonian gas dynamics to relativistic gas dynamics is presented. It is found that the relativistic Euler equations are split into a set of discrete conservation laws with beam split conservative state vectors and flux vectors. Highorder accurate schemes using essentially nonoscillatory concept are devised. Formulations for two space dimensions are also included. Numerical experiments with relativistic one-dimensional shock tube flows and two-dimensional Kelvin-Helmholtz instability flow to illustrate the method are given. (c) 1997 Academic Press
\end{abstract}

\section{INTRODUCTION}

In recent years relativistic gas dynamics plays an important role in areas of astrophysics, high energy particle beams, high energy nuclear collisions, and free-electron laser technology. The equations that describe the relativistic gas dynamics are highly nonlinear and analytical solutions to practical problems are difficult to obtain and numerical solutions are usually pursued. Several numerical methods for solving relativistic gas dynamics have been reported $[1,4,7,14]$ and they are mostly developed out of the existing reliable methods for solving the Euler equations of nonrelativistic or Newtonian gas dynamics. It is also noted that almost all the methods are based on macroscopic continuum description.

In [13] Sanders and Prendergast presented an interesting explicit scheme, which they called the beam scheme, for solving the equilibrium limit of the nonrelativistic Boltzmann equation, namely the the Euler equations of Newtonian gas dynamics. In the beam scheme, a presumed nonrelativistic Maxwellian velocity distribution function is approximated by several Dirac delta functions or discrete beams of particles in each cell. These beams are permitted to move over a time step transporting mass, momentum, and energy into adjacent cells. The motion of each beam is followed to first-order accuracy. The transport is taken into account to determine the new mass, momentum, and energy in each cell; and these macroscopic moments are

\footnotetext{
${ }^{1}$ Author to whom all the correspondence should be addressed. E-mail: yangjy@spring.iam.ntu.edu.tw.
}

used to describe the new local nonrelativistic Maxwellian distribution for each cell. The entire process is then repeated for the next time step. The choice of the size of the time step is dictacted by the Courant-Friedrich-Lewy stability condition that physically no beam of gas travels farther than one cell spacing in one time step. For further details see [13]. The beam scheme, although it is a particle method, however, has all the desirable features of modern characteristic-based wave propagating methods, the socalled upwind shock-capturing methods for hyperbolic conservation laws. Recently, a new hydrodynamic code based on the solution of the BGK Boltzmann equation [2] has been presented by Prendergast and $\mathrm{Xu}[11,20]$. In the method, the Euler and Navier-Stokes solutions are obtained out of the BGK model Boltzmann equation in which both the integral solution of the BGK kinetic model and the collision constraints are used simultaneously. Only the first-order Taylor expansions for the nonequilibrium distribution function and the local Maxwellian have been used although all trajectories of individual particles are followed. Thus their method is of second-order accuracy. Further development and application of their method to practical aerodynamic problems has been reported [21]. The Boltzmann-type Euler scheme of Prendergast and Xu [11] is quite different from the beam scheme. In general, the beam scheme is more dissipative than the Euler method of Prendergast and $\mathrm{Xu}$ as far as a Riemann solver is concerned. Another related Euler method based on the kinetic theory is presented in $[23,24]$ in which the discrete ordinate method is used to solve the Euler limit solution of the Boltzmann equation by replacing the distribution function by the local Maxwellian. The choice of the discrete ordinate is dictated by the requirement that the distribution function and its moments can be accurately represented. Usually, the Gauss-Hermite quadrature and the equally spaced quadrature are used. However, the number of discrete ordinate in the velocity space can become unbearable large in order to accurately represent the distribution function, particularly when the temperature is high. By contrast, only seven beams are needed in the beam scheme even in the three space dimensions but the beam scheme is limited to equilibrium flows only. The three kinetic theory based 
approaches, namely, the beam scheme, the Boltzmanntype Euler/Navier-Stokes solver of Prendergast and $\mathrm{Xu}$ and the discrete ordinate method, seem to give good Euler solutions for Newtonian gas dynamics. Extension of the above three methods to relativistic gas dynamics seems to be feasible. However, the degree of difficulty and the complexity involved may vary for each method and the algebra needs to be worked out becomes more complicated due to the Lorentz factor and the complex function form of the Juttner distribution [8] which make the set of relativistic equations highly nonlinear and stiff.

In this work, we adopt the concept of the beam scheme for Newtonian gas dynamics and directly extend it to relativistic gas dynamics. Although the thinking is straightforward, however, due to the complex function form of the relativistic Maxwellian distribution (involving modified Bessel function), the derivations of the relativistic beam scheme are by no means obvious and some care is needed. A class of high-order relativistic beam schemes are also given. The structure of the paper is organized as follows. In Section 2 elements of relativistic kinetic theory are briefly described. The relativistic Maxwellian, related definitions and conservation laws are given. In Section 3 the firstorder relativistic beam scheme is constructed. High-order nonoscillatory methods are devised in Section 4. Formulations for two space dimensions are derived in Section 5. Numerical experiments to illustrate the relativistic beam scheme are given in Section 6. Relativistic flows in one and two space dimensions are computed. In Section 7 some concluding remarks are included.

\section{ELEMENTS OF RELATIVISTIC KINETIC THEORY}

In this section, we give a brief account of relativistic kientic theory of dilute gas appropriate for present work in content. The works in $[3,9,16-18]$ are found to be useful and should be consulted. Let us consider a simple system consisting of relativisitc particles of mass $m$ with momenta $\mathbf{p}$ and energies $c p^{0}$, where $p^{0}=\left(\mathbf{p}^{2}+m^{2} c^{2}\right)^{1 / 2}$ and $c$ stands for the speed of light. Introduce a function $f(\bar{x}, \bar{p})$ which gives the distribution of the four-momenta $\bar{p}=p^{\mu}=\left(p^{0}, \mathbf{p}\right)$ in each space-time point $\bar{x}=x^{\mu}=(c t$, $\mathbf{x})$. The relativistic Boltzmann transport equation which describes the time evolution of the single particle distribution function $f(\bar{x}, \bar{p})$, is

$$
p^{\mu} \partial_{\mu} f(\bar{x}, \bar{p})=C(\bar{x}, \bar{p})
$$

where $C(\bar{x}, \bar{p})$ is the collision integral term representing changes in $f$ due to particle interactions. The four-momentum is a time-like vector with normalization $\left(p^{0}\right)^{2}-\mathbf{p}^{2}=$ $m^{2} c^{2}$, that is,

$$
p^{\mu} p_{\mu}=m^{2} c^{2}
$$

where $p^{\mu}$ are the contravariant, and $p_{\mu}$ are the covariant components of a four vector. The $p^{\mu}$ and $p_{\mu}$ are related by

$$
p_{\mu}=g_{\mu \nu} p^{\mu}
$$

where

$$
g_{\mu \nu}=g^{\mu \nu}=\operatorname{diag}(1,-1,-1,-1)
$$

is the metric tensor.

The local density $n(\bar{x})$ and the local particle flow $\mathbf{j}(\bar{x})$ are defined by

$$
\begin{aligned}
& n(\bar{x})=\int d^{3} p f(\bar{x}, \bar{p}), \\
& \mathbf{j}(\bar{x})=\int d^{3} p \mathbf{u} f(\bar{x}, \bar{p}),
\end{aligned}
$$

where

$$
\mathbf{u}=c \mathbf{p} / p^{0}=\left(u_{x}, u_{y}, u_{z}\right)
$$

is the three-velocity of a relativistic particle with momentum p. The particle four flow, $N^{\mu}(\bar{x})=(\operatorname{cn}(\bar{x}), \mathbf{j}(\bar{x}))$, is

$$
N^{\mu}(\bar{x})=c \int \frac{d^{3} p}{p^{0}} p^{\mu} f(\bar{x}, \bar{p}) .
$$

The energy-momentum tensor $T^{\mu \nu}(\bar{x})$ is given by

$$
T^{\mu \nu}(\bar{x})=c \int \frac{d^{3} p}{p^{0}} p^{\mu} p^{v} f(\bar{x}, \bar{p})
$$

The hydrodynamic four-velocity of a medium, $U^{\mu}$, is a time-like vector parallel to the world-line of the particles. It is defined as

$$
U^{\mu} U_{\mu}(\bar{x})=c^{2}, U^{\mu}=(\gamma c, \gamma \mathbf{u}), U_{\mu}=(\gamma,-\gamma \mathbf{u}),
$$

where $\gamma=1 / \sqrt{1-\mathbf{u}^{2} / c^{2}}$ is the Lorentz factor. With the help of the hydrodynamic velocity we define a projector, $\Delta^{\mu \nu}$, which projects onto the plane orthogonal to $U^{\mu}$. The orthogonal projector can be constructed as

$$
\Delta^{\mu \nu}=g^{\mu \nu}-\frac{U^{\mu} U^{\nu}}{c^{2}}
$$

The projector is acting on the flow velocity and yields $\Delta^{\mu \nu} U_{\mu}=0$. This projector helps one to find the local rest frame which moves together with the matter. The local rest (LR) frame is the reference frame, where the hydrodynamic velocity has the components 


$$
U_{\mathrm{LR}}^{\mu}=(c, 0,0,0)
$$

In this local rest frame one has

$$
\Delta_{\mathrm{LR}}^{\mu \nu}=\Delta_{\mu \nu \mathrm{LR}}=\operatorname{diag}(0,-1,-1,-1) .
$$

To specify the hydrodynamic velocity we choose the definition due to Eckart, that is,

$$
U^{\mu}=\frac{c N^{\mu}}{\sqrt{N^{\nu} N_{\nu}}}
$$

and we have no particle flow in the local rest frame in the spatial directions

$$
N_{\mathrm{LR}}^{i}=0, \quad i=1,2,3 .
$$

Now we consider the equilibrium limit of the Boltzmann transport equation. It is well known that the effect of collision integral is to drive the gas toward a state of thermodynamic equilibrium specified by a relativistic Maxwellian (or Jüttner) distribution [8]. To solve the equilibrium limit of the relativistic Boltzmann equation is equivalent to set $f=f^{(0)}(\bar{x}, \bar{p})$ in Eq. (2.1), for which the collision integral satisfies $C(\bar{x}, \bar{p})=0$, automatically. Equation (2.1) now becomes

$$
\partial_{t} f^{(0)}(\bar{x}, \bar{p})+\mathbf{u} \cdot \nabla_{\boldsymbol{x}} f^{(0)}(\bar{x}, \bar{p})=0
$$

For equilibrium relativistic gas flow, the distribution function of the gas is the local relativistic Maxwellian

$$
\begin{aligned}
f^{(0)}(\bar{x}, \bar{p})= & n(\bar{x}) \frac{m c^{2} / k_{\mathrm{B}} T}{4 \pi m^{3} c^{3} K_{2}\left(m c^{2} / k_{\mathrm{B}} T\right)} \\
& \exp \left[-\frac{m c^{2}}{k_{\mathrm{B}} T} \sqrt{1+\left(\frac{\mathbf{p}}{m c^{2}}\right)^{2}}\right],
\end{aligned}
$$

where $m$ is the mass of the gas particle, $T$ is the gas temperature, $k_{\mathrm{B}}$ the Boltzmann constant and $K_{\nu}(z)$ is the modified Bessel function of order $\nu$,

$$
K_{\nu}(z)=\frac{(z / 2)^{\nu} \Gamma(1 / 2)}{\Gamma[\nu+1 / 2]} \int_{1}^{\infty} e^{-z t}\left(t^{2}-1\right)^{\nu-1 / 2} d t .
$$

The particle density $n$ and the rest mass density $\rho$ in equilibrium are given by

$$
n(\bar{x})=\frac{1}{c} \int \frac{d^{3} \mathbf{p}}{p^{0}} p^{\mu} U_{\mu} f^{(0)}, \quad \rho(\bar{x})=m n(\bar{x}) .
$$

The energy-momentum tensor in equilibrium is defined by

$$
T^{\mu \nu}=c \int \frac{d^{3} \mathbf{p}}{p^{0}} p^{\mu} p^{\mu} f^{(0)}=\frac{1}{c^{2}}(n \varepsilon+p) U^{\mu} U^{\nu}-p g^{\mu \nu},
$$

where $\varepsilon$ is the average energy per particle and $e$ is the energy density in the local rest frame

$$
e=n \varepsilon=\frac{1}{c} \int \frac{d^{3} \mathbf{p}}{p^{0}}\left(p^{\mu} U_{\mu}\right)^{2} f^{(0)}
$$

and the equilibrium pressure

$$
p=-\frac{1}{3} c \int \frac{d^{3} \mathbf{p}}{p^{0}} p^{\mu} p^{\nu} \Delta_{\mu \nu} f^{(0)} .
$$

After inserting $f(0)$ into the above definitions, we obtain

$$
\varepsilon=m c^{2} \frac{K_{1}\left(m c^{2} / k_{\mathrm{B}} T\right)}{K_{2}\left(m c^{2} / k_{\mathrm{B}} T\right)}+3 k_{\mathrm{B}} T
$$

and we have

$$
p=n k_{\mathrm{B}} T
$$

This is the equation of state of the relativistic perfect gas of classical particles. The conservation equations are obtained from the moment integration of Eq. (2.16) and can be expressed as

$$
\begin{aligned}
\partial_{\mu}\left(\rho U^{\mu}\right) & =0 \\
\partial_{\mu} T^{\mu \nu} & =0 .
\end{aligned}
$$

We first consider relativistic flows in one space dimension. All macroscopic properties are assumed to be functions of $x^{1}=x$ and $x^{0}=c t$ and $u_{x}=v, u_{y}=u_{z}=0$. We also set the speed of light equal to unity, $c \equiv 1$. In one space dimension we consider that a laboratory frame moves at a constant speed $u_{x}=v$ in the $x$-direction with respect to a local rest frame. Any four-vector in the local rest frame, $A_{\mathrm{LR}}^{\mu}$, is related to that in the laboratory frame, $A^{\mu}$, through the Lorentz transformation

$$
\begin{gathered}
L_{1}=\left[\begin{array}{cccc}
\gamma_{v} & \gamma_{v} v & 0 & 0 \\
\gamma_{v} v & \gamma_{v} & 0 & 0 \\
0 & 0 & 1 & 0 \\
0 & 0 & 0 & 1
\end{array}\right], \\
\gamma_{v}=\frac{1}{\sqrt{1-v^{2}}} .
\end{gathered}
$$

Since $U_{\mathrm{LR}}^{\mu}=(c=1,0,0,0)$ and we have $U^{\mu}=L_{1} u_{\mathrm{LR}}^{\mu}=$ $\left(\gamma_{v}, \gamma_{v} v, 0,0\right)$. In the laboratory frame and in one space dimension, the conservation equations Eq. (2.25) and Eq. (2.26) give the following relativistic Euler equations: 


$$
\begin{gathered}
\partial_{t} Q+\partial_{x} F=0 \\
Q=\left(\begin{array}{c}
R \\
M \\
E
\end{array}\right), \quad F=\left(\begin{array}{c}
R v \\
M v+p \\
E v+p v
\end{array}\right) .
\end{gathered}
$$

The laboratory frame quantities $R, M$, and $E$ are related to the quantities in the local rest frame $n$ (or $\rho$ ) and $e$ and to the fluid velocity $v$ by a set of nonlinear transforms

$$
\begin{gathered}
R=\gamma_{v} \rho, \\
M=T^{01}=\gamma_{v}^{2}(e+p) v, \\
E=T^{00}=\gamma_{v}^{2}(e+p)-p .
\end{gathered}
$$

The system of relativistic Euler equations can be closed by specifying an equation of state

$$
p=p(e, \rho)
$$

For gamma-law gas, we have the equation of state as

$$
p=(\Gamma-1)(e-\rho),
$$

where $\Gamma$ is a constant and $1<\Gamma<\frac{5}{3}$.

For given values of $R, M$, and $E$, Eqs. (2.30)-(2.32), with the help of the equation of state, Eq. (2.33) or Eq. (2.34), and the definition of $\gamma_{v}$, Eq. (2.28), provide a set of five equations for five unknowns $n, e, p, v$, and $\gamma_{v}$, which have to be solved in each cell during a time integration step. In the next section, we shall derive a numerical method for solving the relativistic Euler equations, Eq. (2.29), based on the concept of nonrelativistic beam scheme due to Sanders and Prendergast [13].

\section{THE RELATIVISTIC BEAM SCHEME}

In this section the first-order relativistic beam scheme is derived. We consider the one-dimensional relativistic flow problems in which the macroscopic quantities are assumed to be functions of $x^{1}=x$ and $x^{0}=t$. Divide the space into a number of cells of size $\Delta x_{j}=x_{j+1}-x_{j}$. Without loss of generality, we assume that the cells are of equal size $\Delta x$. The local state of the gas in each cell $j$ at any time $t$ is specified by three macrocopic properties, $Q_{j}=\left(R(t)_{j}\right.$, $\left.M(t)_{j}, E(t)_{j}\right)^{\mathrm{T}}$, which are the mass density, momentum density and the energy density in cell $j$, respectively. The fundamental approximation of beam scheme of Sanders and Prendergast [13] for nonrelativistic gas dynamics is to replace the local nonrelativistic Maxwellian by three delta functions or discrete beams of particles which reproduce the appropriate moments of the distribution function. Here in the relativistic gas dynamics, due to the complex func- tional form of the Jüttner distribution function $f^{(0)}$ [8], we first approximate it in a three-dimensional manner by seven delta functions or discrete beams of particles which reproduce the appropriate moments of the distribution function. Eventually, for one space dimension, it will be reduced to three beams. In the laboratory frame we first approximate $f^{(0)}$ in each cell $j$ by

$$
\begin{aligned}
f^{(0)}(\bar{p}) \approx q_{j}(\bar{p})= & a_{j} \delta\left(\bar{p}-\bar{p}_{0}\right)+b_{j} \delta\left(\bar{p}-\bar{p}_{x+}\right) \\
& +b_{j} \delta\left(\bar{p}-\bar{p}_{x^{-}}\right)+b_{j} \delta\left(\bar{p}-\bar{p}_{y^{+}}\right) \\
& +b_{j} \delta\left(\bar{p}-\bar{p}_{y^{-}}\right)+b_{j} \delta\left(\bar{p}-\bar{p}_{z^{+}}\right) \\
& +b_{j} \delta\left(\bar{p}-\bar{p}_{z^{-}}\right) .
\end{aligned}
$$

Such an approximation can be viewed as dividing the particles in each cell into seven groups. If one observes the flow in the local rest frame of the cell, then one would like to have one group is at rest and the other six groups of particles are moving in the $\pm x, \pm y$, and $\pm z$, directions, respectively. Naturally, in each cell $j$, one would like to have the speed for each of the six groups to be $\Delta v$ and with same weight $b_{j}$ and the one group left would have weight $a_{j}$. Consider the one-dimensional problem first, the macroscopic velocity is $(v, 0,0)$. The Lorentz transformation between the rest frame and the lab frame is given by Eq. (2.27). By using this Lorentz transformation we can obtain $\bar{p}_{x \pm}, \bar{p}_{y \pm}, \bar{p}_{z^{ \pm}}$, and $\bar{p}_{0}$ as follows:

$$
\begin{gathered}
\bar{p}_{x \pm}=L_{1}\left(\begin{array}{c}
\gamma_{\Delta} m \\
\pm \gamma_{\Delta} m \Delta v \\
0 \\
0
\end{array}\right), \quad \bar{p}_{y \pm}=L_{1}\left(\begin{array}{c}
\gamma_{\Delta} m \\
0 \\
\pm \gamma_{\Delta} m \Delta v \\
0
\end{array}\right), \\
\bar{p}_{z \pm}=L_{1}\left(\begin{array}{c}
\gamma_{\Delta} m \\
0 \\
0 \\
\pm \gamma_{\Delta} m \Delta v
\end{array}\right), \quad \bar{p}_{0}=L_{1}\left(\begin{array}{c}
m \\
0 \\
0 \\
0
\end{array}\right)
\end{gathered}
$$

where $\gamma_{\Delta}=1 /\left(1-\Delta v^{2}\right)^{1 / 2}$ and $\Delta v$ is to be determined later. Thus, the unknown parameters in each cell $j$ in Eq. (3.1) are $a_{j}, b_{j}$, and $(\Delta v)_{j}$, and they are determined in such a way that the following quantities in the local rest frame are preserved:

$$
\begin{gathered}
R_{j}=\gamma_{v} \rho_{j}=\int \gamma_{v} \rho_{j} q_{j}(\bar{p}) d^{3} p \\
M_{j}=T_{j}^{01}=\gamma_{v}^{2}\left(e_{j}+p_{j}\right) v_{j}=\int \rho_{j} \gamma_{v}^{2} v_{j} q_{j}(\bar{p}) d^{3} p \\
E_{j}=T_{j}^{00}=\gamma_{v}^{2}\left(e_{j}+p_{j}\right)-p_{j}=\int \gamma_{v}^{2} \rho_{j} q_{j}(\bar{p}) d^{3} p
\end{gathered}
$$


Then we obtain the equations for the determination of the parameters $\left(a_{j}, b_{j}, \Delta v\right)$ as

$$
\begin{gathered}
a_{j}+6 b_{j} \gamma_{\Delta j}=1, \\
2 b_{j} \gamma_{\Delta j}^{2} \Delta v^{2}=p_{j} / \rho_{j}, \\
a_{j}+6 b_{j} \gamma_{\Delta j}^{2}=e_{j} / \rho_{j} .
\end{gathered}
$$

These give

$$
\begin{aligned}
\gamma_{\Delta j} & =\frac{e_{j}-\rho_{j}}{3 p_{j}-\left(e_{j}-\rho_{j}\right)}, \\
\Delta v & =\sqrt{\gamma_{\Delta j}^{2}-1} / \gamma_{\Delta j}, \\
b_{j} & =\frac{\left(e_{j} / \rho_{j}-1\right)}{6 \gamma_{\Delta j}\left(\gamma_{\Delta j}-1\right)}, \\
a_{j} & =1-6 b_{j} \gamma_{\Delta j} .
\end{aligned}
$$

It is noted that we require

$$
\gamma_{\Delta j}>1, \quad a_{j}>0, \quad b_{j}>0 .
$$

Define the following quantities:

$$
\begin{gathered}
v_{ \pm}=\frac{v \pm \Delta v}{1 \pm v \Delta v}, \\
\gamma_{ \pm}=\frac{1}{\sqrt{1-v_{ \pm}^{2}}}, \quad \gamma_{+}+\gamma_{-}=2 \gamma_{v} \gamma_{\Delta} .
\end{gathered}
$$

After obtaining the parameters $a, b$, and $\Delta v$, we can determine the conservative quantities carried by each beam as

$$
\begin{aligned}
R_{\sigma, j} & =\int \gamma \rho_{j} c_{\sigma, j} \delta\left(\bar{p}-\bar{p}_{\sigma, j}\right) d^{3} p, \\
M_{x, \sigma, j} & =\int \gamma^{2} \rho_{j} u_{x} c_{\sigma, j} \delta\left(\bar{p}-\bar{p}_{\sigma, j}\right) d^{3} p, \\
M_{y, \sigma, j} & =\int \gamma^{2} \rho_{j} u_{y} c_{\sigma, j} \delta\left(\bar{p}-\bar{p}_{\sigma, j}\right) d^{3} p, \\
M_{z, \sigma, j} & =\int \gamma^{2} \rho_{j} u_{z} c_{\sigma, j} \delta\left(\bar{p}-\bar{p}_{\sigma, j}\right) d^{3} p, \\
E_{\sigma, j} & =\int \gamma^{2} \rho_{j} c_{\sigma, j} \delta\left(\bar{p}-\bar{p}_{\sigma, j}\right) d^{3} p,
\end{aligned}
$$

where $\bar{p}_{\sigma, j}$ denote $\bar{p}_{0, j}, \bar{p}_{x \pm, j}, \bar{p}_{y \pm, j}$ and $\bar{p}_{z \pm, j}$, respectively, and

$$
c_{\sigma, j}= \begin{cases}a_{j}, & \sigma=1, \\ b_{j}, & \sigma=2,3, \ldots, 7 .\end{cases}
$$

In the laboratory frame, the contribution of each $\sigma$ beam to the conservative state vector $Q$ in cell $j$ is

$$
Q_{\sigma, j}=\left(\begin{array}{c}
m_{\sigma, j} U_{\sigma, j}^{0} \\
m_{\sigma, j} U_{\sigma, j}^{0} U_{\sigma, j}^{1} \\
m_{\sigma, j} U_{\sigma, j}^{0} U_{\sigma, j}^{2} \\
m_{\sigma, j} U_{\sigma, j}^{0} U_{\sigma, j}^{3} \\
m_{\sigma, j} U_{\sigma, j}^{0} U_{\sigma, j}^{0}
\end{array}\right),
$$

where $\bar{U}_{\sigma, j}=\left(U_{\sigma}^{0}, U_{\sigma}^{1}, U_{\sigma}^{2}, U_{\sigma}^{3}\right)$ is the velocity four-vector of each beam $\sigma$ in the laboratory frame

$$
\begin{gathered}
\bar{U}_{1}=\left(\gamma_{v}, \gamma_{v} v, 0,0\right), \quad \bar{U}_{2}=\left(\gamma_{+}, \gamma_{+} v_{+}, 0,0\right), \\
\bar{U}_{3}=\left(\gamma_{-}, \gamma_{-} v_{-}, 0,0\right), \\
\bar{U}_{4}=\left(\gamma_{v} \gamma_{\Delta}, \gamma_{v} \gamma_{\Delta} v, \gamma_{\Delta} \Delta v, 0\right), \quad \bar{U}_{5}=\left(\gamma_{v} \gamma_{\Delta}, \gamma_{v} \gamma_{\Delta} v,-\gamma_{\Delta} \Delta v, 0\right), \\
\bar{U}_{6}=\left(\gamma_{v} \gamma_{\Delta}, \gamma_{v} \gamma_{\Delta} v, 0, \gamma_{\Delta} \Delta v\right), \quad \bar{U}_{7}=\left(\gamma_{v} \gamma_{\Delta}, \gamma_{v} \gamma_{\Delta} v, 0,-\gamma_{\Delta} \Delta v\right) .
\end{gathered}
$$

Now define the $x$ velocity of each beam, $V_{\sigma, j}=U_{\sigma, j}^{1} / U_{\sigma, j}^{0}$, $(\sigma=1,2, \ldots, 7)$ in cell $j$ as

$$
\begin{aligned}
& V_{1, j}=v_{j}=V_{4, j}=V_{5, j}=V_{6, j}=V_{7, j}, \\
& V_{2, j}=v_{+j}, \quad V_{3, j}=v_{-j},
\end{aligned}
$$

and the mass carried by each beam as

$$
m_{1, j}=a_{j} \rho_{j} \Delta x, \quad m_{\sigma, j}=b_{j} \rho_{j} \Delta x \quad(\sigma=2,3, \ldots, 7) .
$$

It is noted that $\sum_{\sigma=1}^{7} Q_{\sigma, j}=\Delta x Q_{j}$. During an interval of time $\Delta t$, these discrete beams will move and transfer mass, momentum, and energy into adjacent cells. The time step $\Delta t$, is subjected to the condition that no single beam moves farther than a cell width $\Delta x$ during $\Delta t$, i.e.,

$$
\Delta t \leq \frac{\Delta x}{\max \left(\left|V_{\sigma, j}\right|\right)},
$$

which is the Courant-Friedrich-Lewy stability condition. During time step $\Delta t$ beam $\sigma(\sigma=1,2, \ldots, 7)$ in cell $j$ moves a distance either into cell $j-1$ or cell $j+1$ or remains in cell $j$ depending on the sign of the beam velocities. Define the fraction that transfers from cell $j$ to cells $k=j \pm 1$ as

$$
\begin{aligned}
\alpha_{\sigma, j, j \pm 1}= \pm \frac{1}{2}\left(V_{\sigma, j} \pm\left|V_{\sigma, j}\right|\right) \frac{\Delta t}{\Delta x} & = \pm V_{\sigma, j}^{ \pm} \lambda, \\
\lambda & =\frac{\Delta t}{\Delta x},
\end{aligned}
$$

and the fraction that remains in cell $j$ is

$$
\alpha_{\sigma, j, j}=1-\left|V_{\sigma, j}\right| \lambda
$$


After time step $\Delta t$, the new values of mass, momentum, and energy in each cell, $\left(Q_{j}^{n+1}\right)$, taking into account transfer of these quantities from adjacent cells are given by

$$
Q_{j}^{n+1}=\frac{1}{\Delta x} \sum_{\sigma=1}^{7} \sum_{k=j-1}^{j+1} \alpha_{\sigma, k, j}^{n} Q_{\sigma, k}^{n},
$$

where

$$
Q_{j}^{n+1}=\left(\begin{array}{c}
\gamma_{v, j} \rho_{j} \\
\gamma_{v, j}^{2}\left(e_{j}+p_{j}\right) v_{j} \\
0 \\
0 \\
\gamma_{v, j}^{2}\left(e_{j}+p_{j}\right)-p_{j}
\end{array}\right)
$$

The integration scheme, Eq. (3.22), can be expressed as

$$
\begin{aligned}
Q_{j}^{n+1}= & \frac{1}{\Delta x} \sum_{\sigma=1}^{7}\left[\alpha_{\sigma, j-1, j} Q_{\sigma, j-1}^{n}+\alpha_{\sigma, j, j} Q_{\sigma, j}^{n}\right. \\
& \left.+\alpha_{\sigma, j+1, j} Q_{\sigma, j+1}^{n}\right] \\
= & \frac{1}{\Delta x} \sum_{\sigma=1}^{7}\left[V_{\sigma, j-1}^{+} Q_{\sigma, j-1} \lambda+\left(1-\left|V_{\sigma, j}^{n}\right| \lambda\right) Q_{\sigma, j}\right. \\
& \left.+V_{\sigma, j+1}^{-} Q_{\sigma, j+1} \lambda\right] .
\end{aligned}
$$

For problems in one space dimension, the seven beams can be merged into three beams at this stage. Since $V_{1, j}=$ $V_{4, j}=V_{5, j}=V_{6, j}=V_{7, j}=v_{j}$, we can combine these beams together and form a new single beam

$$
\tilde{Q}_{1, j}=Q_{1, j}+Q_{4, j}+Q_{5, j}+Q_{6, j}+Q_{7, j}
$$

The other two beams are kept unchanged

$$
\begin{aligned}
& \tilde{Q}_{2, j}=Q_{2, j}, \\
& \tilde{Q}_{3, j}=Q_{3, j} .
\end{aligned}
$$

The corresponding new three beam velocities $\tilde{V}_{\sigma}$ at cell $j$ are

$$
\tilde{V}_{1, j}=v_{j}, \quad \tilde{V}_{2, j}=v_{+, j}, \quad \tilde{V}_{3, j}=v_{-, j}
$$

We then have the beam flux vectors for the three new beams

$$
\tilde{F}_{\sigma, j}=\tilde{V}_{\sigma, j} \tilde{Q}_{\sigma, j}, \quad \sigma=1,2,3
$$

From here on, without causing any confusion we shall omit the tilde signs in the equations to be described below. We also reset the number of beams from seven to three, i.e., $\sigma=1,2,3$. The above integration scheme, Eq. (3.24), can also be cast into the form of a conservative scheme in terms of numerical fluxes

$$
\begin{aligned}
& Q_{j}^{n+1}=\frac{1}{\Delta x} \sum_{\sigma=1}^{3} Q_{\sigma}^{n+1}, \\
& Q_{\sigma}^{n+1}=Q_{\sigma, j}^{n}-\frac{\Delta t}{\Delta x}\left(\tilde{F}_{\sigma, j+1 / 2}^{\mathrm{SP}}-\tilde{F}_{\sigma, j-1 / 2}^{\mathrm{SP}}\right),
\end{aligned}
$$

where the numerical flux for each beam, $\tilde{F}_{\sigma, j+1 / 2}^{\mathrm{SP}}$, is defined as

$$
\begin{gathered}
\tilde{F}_{\sigma, j+1 / 2}^{\mathrm{SP}}=\frac{1}{2}\left(F_{\sigma, j}^{n}+F_{\sigma, j+1}^{n}-\Phi_{\sigma, j+1 / 2}^{\mathrm{SP}}\right), \\
\Phi_{\sigma, j+1 / 2}^{\mathrm{SP}}=\left|V_{\sigma, j+1}\right| Q_{\sigma, j+1}-\left|V_{\sigma, j}\right| Q_{\sigma, j} .
\end{gathered}
$$

This completes the description of the first-order relativistic beam scheme.

The scheme defined by Eqs. (3.28)-(3.30) can be considered as a conservative upwind scheme for the following discrete set of conservation laws

$$
\partial_{t} Q_{\sigma}+\partial_{x} F_{\sigma}\left(Q_{\sigma}\right)=0, \quad \sigma=1,2,3
$$

with

$$
\begin{aligned}
Q_{1} & =\left(\begin{array}{c}
\beta\left(a+4 b \gamma_{\Delta}\right) \\
\beta \gamma_{v} v\left(a+4 b \gamma_{\Delta}^{2}\right) \\
\beta \gamma_{v}\left(a+4 b \gamma_{\Delta}^{2}\right)
\end{array}\right), \quad Q_{2}=\left(\begin{array}{c}
\beta_{+} \\
\beta_{+} \gamma_{+} v_{+} \\
\beta_{+} \gamma_{+}
\end{array}\right), \\
Q_{3} & =\left(\begin{array}{c}
\beta_{-} \\
\beta_{-} \gamma_{-} v_{-} \\
\beta_{-} \gamma_{-}
\end{array}\right),
\end{aligned}
$$

and

$$
\begin{aligned}
F_{1} & =\left(\begin{array}{c}
\beta v\left(a+4 b \gamma_{\Delta}\right) \\
\beta \gamma_{v} v^{2}\left(a+4 b \gamma_{\Delta}^{2}\right) \\
\beta \gamma_{v} v\left(a+4 b \gamma_{\Delta}^{2}\right)
\end{array}\right), \quad F_{2}=\left(\begin{array}{c}
\beta_{+} v_{+} \\
\beta_{+} \gamma_{+} v_{+}^{2} \\
\beta_{+} \gamma_{+} v_{+}
\end{array}\right), \\
F_{3} & =\left(\begin{array}{c}
\beta_{-} v_{-} \\
\beta_{-} \gamma_{-} v_{-}^{2} \\
\beta_{-} \gamma_{-} v_{-}
\end{array}\right),
\end{aligned}
$$


where $\beta=\rho \gamma_{v}, \beta_{+}=b \rho \gamma_{+}$, and $\beta_{-}=b \rho \gamma_{-}$. We note that the beam scheme not only splits the state vector but also the flux vectors. It has some entropy-satisfying mechanism embedded as compared with approximate relativistic Riemann solver [4, 14] based on Roe method [12]. Numerical test using first-order beam scheme and Roe-type scheme will be given below to illustrate this point.

After obtaining the new values of the macroscopic gas variables in the laboratory frame $Q_{j}^{n+1}=\left(R_{j}, M_{j}, E_{j}\right)^{n+1}$ for each cell from Eq. (3.28), we need to calculate the local rest frame quantities $\rho_{j}, e_{j}, p_{j}$, and $v_{j}$ at time level $(n+1) \Delta t$. The laboratory quantities $R$ (mass density), $M$ (momentum density), and $E$ (energy density) are related to quantities in the local rest frame $e$ (energy density) and $\rho$ (particle density), and to the fluid velocity $v$ by the set of nonlinear transformations, Eqs. (2.30)-(2.32). From Eqs. (2.30)(2.32) we have the equation for finding $\rho$,

$$
h(\rho)=R^{1}\left(1-\frac{M^{2}}{(E+p(\rho))^{2}}\right)-\rho^{2}=0,
$$

where

$$
p=p(e, \rho)=p\left(E-\sqrt{M^{2}\left(1-\rho^{2} / R^{2}\right)}, \rho\right)=p(\rho) .
$$

A Newton-Raphson procedure can be applied to solve for $\rho$. Once the value of $\rho$ has been obtained, the energy density, pressure, and velocity are obtained via

$$
\begin{gathered}
e=E-\sqrt{M^{2}\left(1-\rho^{2} / R^{2}\right)}, \\
p=p(e, \rho), \\
v=\frac{M}{E+p(e, \rho)} .
\end{gathered}
$$

After the decoding and obtaining the local rest frame quantities, one then specifies that the local relativistic Maxwellian distribution with parameters determined by the new values of the gas variables and repeats the entire procedures to advance the solution in time.

\section{HIGH-ORDER RELATIVISTIC BEAM SCHEMES}

The above scheme is only of first-order accuracy and it is more desirable to have at least second-order accuracy in practical applications. To extend the first-order beam scheme to higher-order accuracy we follow the same approach developed in $[22,23]$ in which the essentially nonoscillatory (ENO) piecewise polynomial reconstruction schemes of Harten et al. [6] were adopted and extended to the Euler equations of Newtonian gas dynamics. Define $\beta_{\sigma, j+1 / 2}^{l}$ as components of $Q_{\sigma, j+1}-Q_{\sigma, j}$. A class of highorder accurate schemes can be expressed in the same form as Eq. (3.29) with the numerical flux defined by

$$
\tilde{F}_{\sigma, j+1 / 2}^{\mathrm{ENO}}=\frac{1}{2}\left(F_{\sigma, j}^{n}+F_{\sigma, j+1}^{n}-\Phi_{\sigma, j+1}^{\mathrm{SP}}-\Phi_{\sigma, j+1 / 2}^{\mathrm{ENO}}\right) .
$$

The components of $\Phi_{\sigma, j+1 / 2}^{\mathrm{ENO}}$ are defined by

$$
\begin{gathered}
\phi_{\sigma, j+1 / 2}^{l \mathrm{ENO}}=\left|\gamma_{\sigma, j+1 / 2}^{l}+\omega \tilde{\gamma}_{\sigma, j+1 / 2}^{l}\right| \beta_{\sigma, j+1 / 2}^{l} \\
-\psi\left(\bar{a}_{\sigma, j+1 / 2}\right)\left(e_{\sigma, j}+e_{\sigma, j+1}\right) \\
-\omega \tilde{\psi}\left(\bar{a}_{\sigma, j+1 / 2}\right)\left(d_{\sigma, j}+d_{\sigma, j+1}\right), \\
\bar{a}_{\sigma, j+1 / 2}^{l}=\bar{a}^{l}\left(Q_{\sigma, j}^{n}, Q_{\sigma, j+1}^{n}\right)= \begin{cases}\frac{F_{\sigma, j+1}^{l}-F_{\sigma, j}^{l}}{\beta_{\sigma, j+1 / 2}^{l},} & \text { if } Q_{\sigma, j}^{l} \neq Q_{\sigma, j+1}^{l}, \\
V_{\sigma, j}, & \text { if } Q_{\sigma, j}^{l}=Q_{\sigma, j+1}^{l} ;\end{cases} \\
e_{\sigma, j}^{l}=\tilde{m}_{\tilde{m}\left[\beta_{\sigma, j+1 / 2}^{l}-\theta \bar{m}\left(\Delta_{-} \beta_{\sigma, j+1 / 2}^{l}, \Delta_{+} \beta_{\sigma, j+1 / 2}^{l}\right),\right.} \\
\left.\beta_{\sigma, j-1 / 2}^{l}+\theta \bar{m}\left(\Delta_{-} \beta_{\sigma, j-1 / 2}^{l}, \Delta_{+} \beta_{\sigma, j-1 / 2}^{l}\right)\right] ; \\
d_{\sigma, j}^{l}= \begin{cases}\bar{m}\left(\Delta_{-} \beta_{\sigma, j-1 / 2}^{l}, \Delta_{+} \beta_{\sigma, j-1 / 2}^{l}\right), & \text { if }\left|\beta_{\sigma, j-1 / 2}^{l}\right| \leq\left|\beta_{\sigma, j+1 / 2}^{l}\right|, \\
\bar{m}\left(\Delta_{-} \beta_{\sigma, j+1 / 2}^{l}, \Delta_{+} \beta_{\sigma, j+1 / 2}^{l}\right), & \text { if }\left|\beta_{\sigma, j-1 / 2}^{l}\right|>\left|\beta_{\sigma, j+1 / 2}^{l}\right| ;\end{cases}
\end{gathered}
$$

$\gamma_{\sigma, j+1 / 2}^{l}= \begin{cases}\psi\left(a_{\sigma, j+1 / 2}^{l}\right)\left(e_{\sigma, j+1}^{l}-e_{\sigma, j}^{l}\right) / \beta_{\sigma, j+1 / 2}^{l}, & \text { if } \beta_{\sigma, j+1 / 2}^{l} \neq 0, \\ 0, & \text { if otherwise }\end{cases}$

$$
\tilde{\gamma}_{\sigma, j+1 / 2}^{l}= \begin{cases}\tilde{\psi}\left(a_{\sigma, j+1 / 2}^{l}\right)\left(d_{\sigma, j+1}^{l}-d_{\sigma, j}^{l}\right) / \beta_{\sigma, j+1 / 2}^{l}, & \text { if } \beta_{\sigma, j+1 / 2}^{l} \neq 0, \\ 0, & \text { if otherwise }\end{cases}
$$

where $\psi$ and $\tilde{\psi}$ functions are defined by

$$
\begin{gathered}
\psi(z)=\left(|z|-\lambda z^{2}\right) / 2, \\
\tilde{\psi}(z)= \begin{cases}\left(2|z|-3 \lambda|z|^{2}+\lambda^{2}|z|^{3}\right) / 6, & \text { if }\left|\beta_{\sigma, j-1 / 2}^{l}\right| \leq\left|\beta_{\sigma, j+1 / 2}^{l}\right|, \\
\left(\lambda^{2}|z|^{3}-|z|\right) / 6, & \text { if }\left|\beta_{\sigma, j-1 / 2}^{l}\right|>\left|\beta_{\sigma, j+1 / 2}^{l}\right| .\end{cases}
\end{gathered}
$$

The $\tilde{m}$ and $\bar{m}$ functions are given by

$$
\begin{aligned}
& \tilde{m}(y, z)= \begin{cases}s \cdot \min (|y|,|z|), & \text { if } \operatorname{sgn} y=\operatorname{sgn} z=x, \\
0, & \text { if otherwise. }\end{cases} \\
& \tilde{m}(y, z)= \begin{cases}y, & \text { if }|y| \leq|z|, \\
z, & \text { if }|y|>|z| .\end{cases}
\end{aligned}
$$

The class of schemes defined by Eqs. (4.2)-(4.11) includes the second-order total variation diminishing (TVD) [5] scheme $(\omega=0, \theta=0)$, denoted as TVD2; the second- 

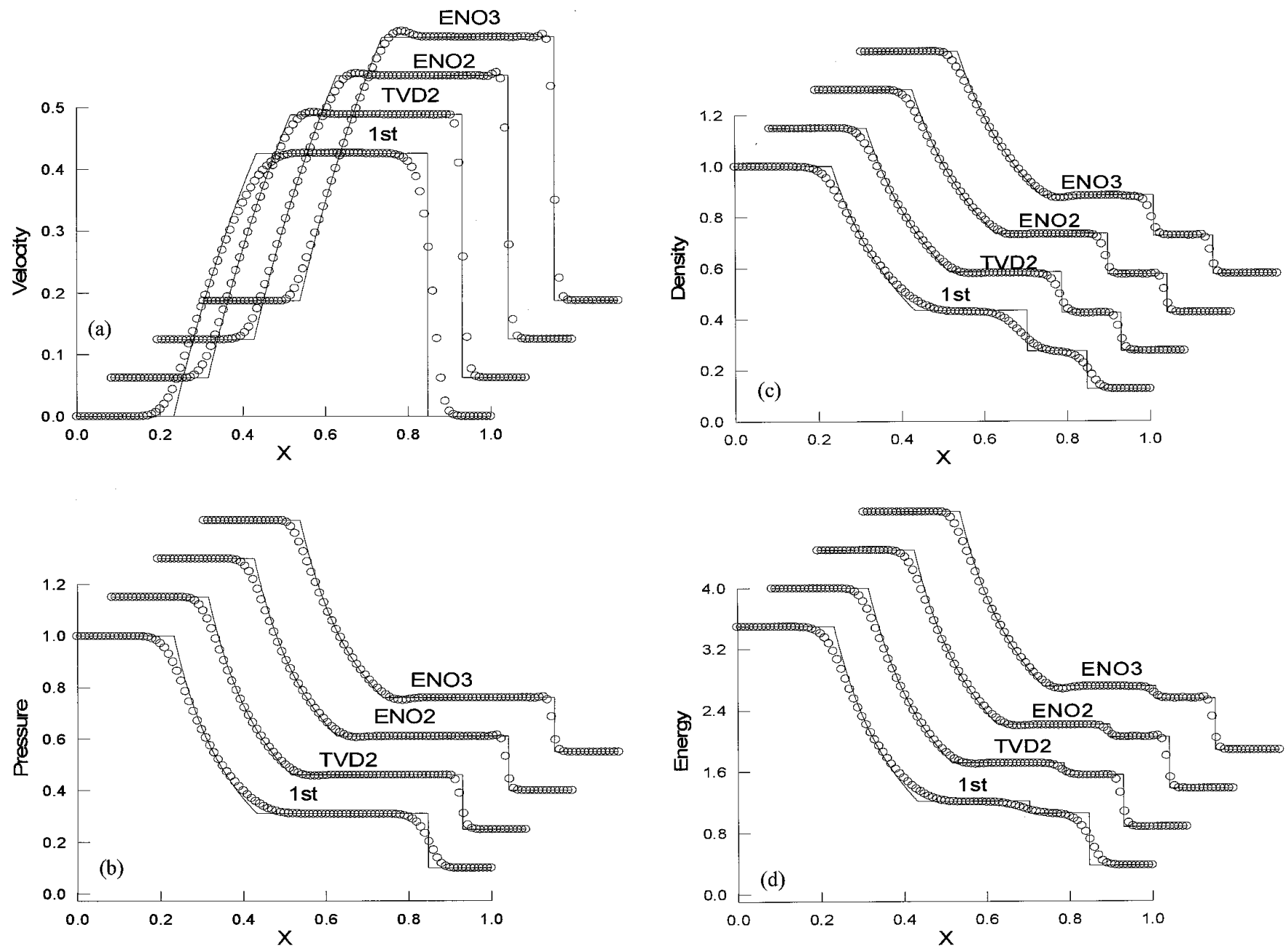

FIG. 1. Relativistic shock tube solution (Example 1), using first-order and high-order beam schemes: (a) velocity; (b) pressure; (c) density; (d) internal energy.

order ENO scheme $(\omega=0, \theta=1 / 2)$, denoted as ENO2; and the third-order UNO scheme $(\omega=1, \theta=0)$, denoted as ENO3 [6, 22]. The details and definitions of the expressions can be found in $[22,23]$.

\section{FORMULATION IN TWO SPACE DIMENSIONS}

In this section we consider relativistic beam scheme in two space dimensions. All macroscopic properties are assumed to be functions of $x^{1}=x, x^{2}=y$, and $x^{0}=t$ and $u^{1}=u_{x}, u^{2}=u_{y}, u^{3}=0$. The Lorentz transformation is

$$
L_{2}=\left[\begin{array}{cccc}
\gamma_{u} & \gamma_{u} u_{x} & \gamma_{u} u_{y} & 0 \\
\gamma_{u} u_{x} & \left(\gamma_{u} u_{x}^{2}+u_{y}^{2}\right) / u^{2} & \left(\gamma_{u}-1\right) u_{x} u_{y} / u^{2} & 0 \\
\gamma_{u} u_{y} & \left(\gamma_{u}-1\right) u_{x} u_{y} / u^{2} & \left(u_{x}^{2}+\gamma_{u} u_{y}^{2}\right) / u^{2} & 0 \\
0 & 0 & 0 & 1
\end{array}\right]
$$

where $\gamma_{u}$ is the Lorentz factor

$$
\gamma_{u}=\frac{1}{\sqrt{1-u^{2}}}, \quad u^{2}=u_{x}^{2}+u_{y}^{2}
$$

Since $U_{\mathrm{LR}}^{\mu}=(c=1,0,0,0)$ and we have $U^{\mu}=L_{2} U_{\mathrm{LR}}^{\mu}=$ $\left(\gamma_{u}, \gamma_{u} u_{x}, \gamma_{u} u_{y}, 0\right)$. In the laboratory frame and in two space dimensions, the conservation equations give the following relativistic Euler equations:

$$
\partial_{t} Q+\partial_{x} F+\partial_{y} G=0,
$$

$$
Q=\left(\begin{array}{c}
R \\
M \\
N \\
E
\end{array}\right), \quad F=\left(\begin{array}{c}
R u_{x} \\
M u_{x}+p \\
N u_{x} \\
E u_{x}+p u_{x}
\end{array}\right), \quad G=\left(\begin{array}{c}
R u_{y} \\
M u_{y} \\
N u_{y}+p \\
E u_{y}+p u_{y}
\end{array}\right)
$$



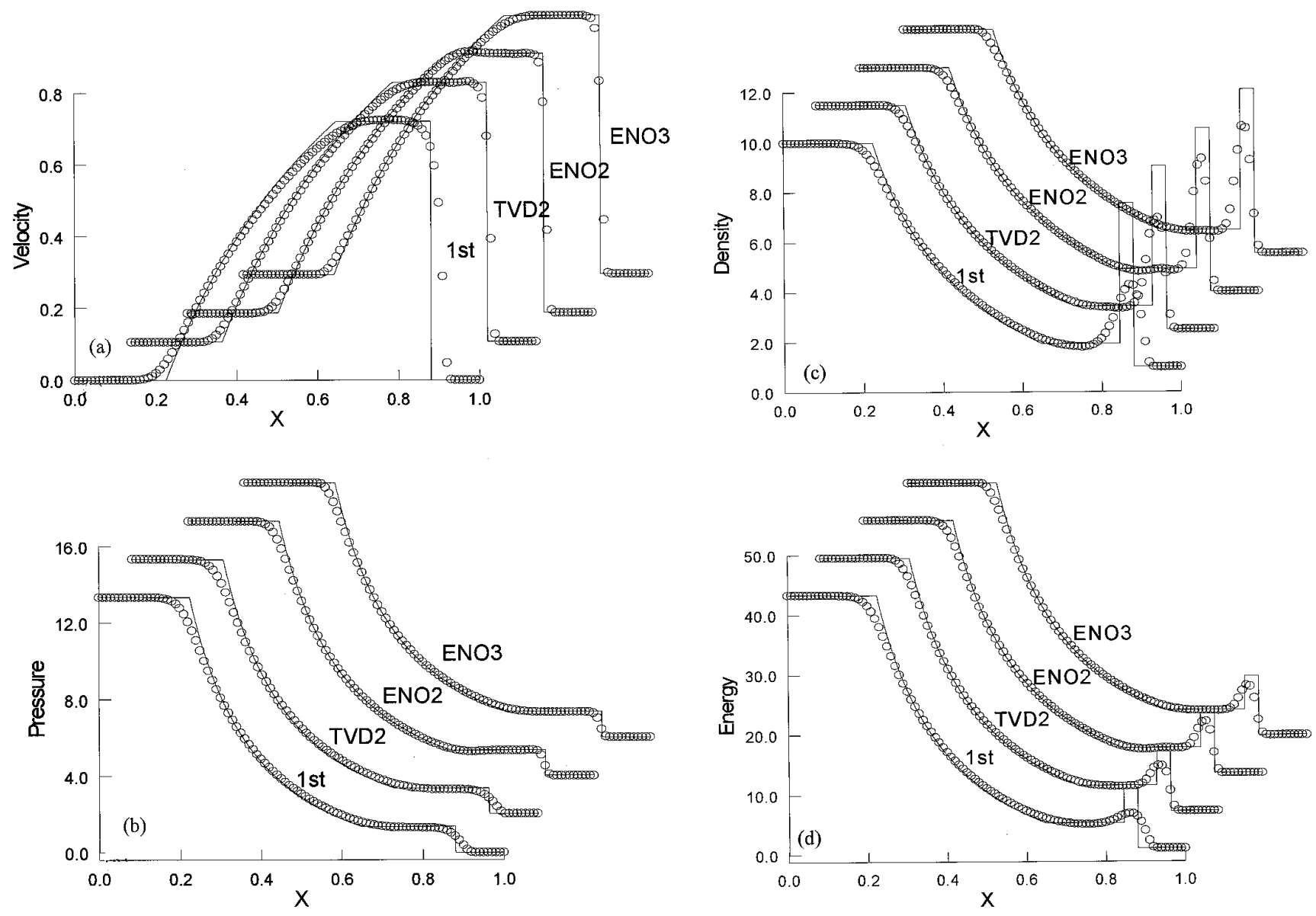

FIG. 2. Relativistic shock tube solution (Example 2), using first-order and high-order beam schemes: (a) velocity; (b) pressure; (c) density; (d) energy.

The laboratory frame quantities $R, M, N$, and $E$ are related to the quantities in the local rest frame $n$ (or $\rho$ ) and $e$ and to the fluid velocities $u_{x}$ and $u_{y}$ by a set of nonlinear transforms

$$
\begin{gathered}
R=\gamma_{u} \rho, \\
M=T^{01}=\gamma_{u}^{2}(e+p) u_{x}, \\
N=T^{02}=\gamma_{u}^{2}(e+p) u_{y}, \\
E=T^{00}=\gamma_{u}^{2}(e+p)-p .
\end{gathered}
$$

The system of relativistic Euler equations can be closed by specifying an equation of state, such as Eq. (2.33).

Next the first-order relativistic beam scheme in two space dimensions is derived. Divide the space into a number of cells of area $\Delta A_{i, j}$. Without loss of generality, we assume uniform rectangular cells with $\Delta x=\Delta y$ and $\Delta A_{i, j}=\Delta x \Delta y$. The local state of the gas in each cell $(i, j)$ at any time $t$ is specified by four macrocopic properties, $Q_{i, j}=(R(t), M(t)$, $N(t), E(t))_{i, j}^{\mathrm{T}}$, which are the mass density, $x$ - and $y$-momen- tum densities and the energy density, respectively. Using Eq. (3.1), we approximate the Juttner distribution function $f^{(0)}$ by seven delta functions or discrete beams of particles which reproduce the appropriate moments of the distribution function. Eventually, for two space dimensions, it can be merged into five beams. In the laboratory frame we first approximate $f^{(0)}$ in each cell $(i, j)$ by

$$
\begin{aligned}
f^{(0)} \approx q_{i, j}(\bar{p})= & a_{i, j} \delta\left(\bar{p}-\bar{p}_{0}\right)+b_{i, j} \delta\left(\bar{p}-\bar{p}_{x+}\right) \\
& +b_{i, j} \delta\left(\bar{p}-\bar{p}_{x-}\right) \\
& +b_{i, j} \delta\left(\bar{p}-\bar{p}_{y+}\right)+b_{i, j} \delta\left(\bar{p}-\bar{p}_{y^{-}}\right) \\
& +b_{i, j} \delta\left(\bar{p}-\bar{p}_{z^{+}}\right)+b_{i, j} \delta\left(\bar{p}-\bar{p}_{z^{-}}\right),
\end{aligned}
$$

where $\bar{p}_{0}, \bar{p}_{x \pm}$, etc. are the same as those defined by Eq. (3.2) and the parameters of $q_{i, j}(\bar{p})$ are $\left(a_{i, j}, b_{i, j},(\Delta v)_{i, j}\right)$ in cell $(i, j)$ and they are determined in such a way that the following quantities in the local rest frame are preserved: 


$$
\begin{gathered}
R_{i, j}=\left(\gamma_{u}\right)_{i, j} \rho_{i, j}=\int \rho_{i, j} \gamma_{u} q_{i, j}(\bar{p}) d^{3} p \\
E_{i, j}=T_{i, j}^{00}=\int \gamma_{u}^{2} \rho_{i, j} q_{i, j}(\bar{p}) d^{3} p \\
\left(M_{x}\right)_{i, j}=T_{i, j}^{01}=\int \gamma_{u}^{2} \rho_{i, j} u_{x} q_{i, j}(\bar{p}) d^{3} p \\
\left(M_{y}\right)_{i, j}=T_{i, j}^{02}=\int \gamma_{u}^{2} \rho_{i, j} u_{y} q_{i, j}(\bar{p}) d^{3} p \\
\left(M_{z}\right)_{i, j}=T_{i, j}^{03}=\int \gamma_{u}^{2} \rho_{i, j} u_{z} q_{i, j}(\bar{p}) d^{3} p=0 .
\end{gathered}
$$

Then we obtain the equations for the determination of the parameters $\left(a_{i, j}, b_{i, j}, \Delta v\right)$, which turn out to be identical to Eq. (3.6) and the solutions are given by Eq. (3.7).

Denote any four-vector $\bar{U}_{\sigma \mathrm{LR}}=\left(U_{\sigma 0}, U_{\sigma 1}, U_{\sigma 2}, U_{\sigma 3}\right)_{\mathrm{LR}}$ in the local rest frame and let

$\bar{U}_{1 \mathrm{LR}}=(1,0,0,0), \quad \bar{U}_{2 \mathrm{LR}}=\left(\gamma_{\Delta}, \gamma_{\Delta} \Delta v, 0,0\right)_{\mathrm{LR}}$,

$\bar{U}_{3 \mathrm{LR}}=\left(\gamma_{\Delta},-\gamma_{\Delta} \Delta v, 0,0\right)_{\mathrm{LR}}$,

$\bar{U}_{4 \mathrm{LR}}=\left(\gamma_{\Delta}, 0, \gamma_{\Delta} \Delta v, 0\right)_{\mathrm{LR}}, \quad \bar{U}_{5 \mathrm{LR}}=\left(\gamma_{\Delta}, 0,-\gamma_{\Delta} \Delta v, 0\right)_{\mathrm{LR}}$,

$\bar{U}_{6 \mathrm{LR}}=\left(\gamma_{\Delta}, 0,0, \gamma_{\Delta} \Delta v\right)_{\mathrm{LR}}, \quad \bar{U}_{7 \mathrm{LR}}=\left(\gamma_{\Delta}, 0,0,-\gamma_{\Delta} \Delta v\right)_{\mathrm{LR}}$.

Then the corresponding four-velocity vectors $\bar{U}_{\sigma}$ in the laboratory frame are given by

$$
\bar{U}_{\sigma}=\left(U_{\sigma 0}, U_{\sigma 1}, U_{\sigma 2}, U_{\sigma 3}\right)=L_{2} \bar{U}_{\sigma \mathrm{LR}} .
$$

Now define the beam velocities

$$
\begin{gathered}
\mathbf{V}_{\sigma}=\left(V_{\sigma x}, V_{\sigma y}, V_{\sigma z}\right)=\left(U_{\sigma 1}, U_{\sigma 2}, U_{\sigma 3}\right) / U_{\sigma 0} \\
(\sigma=1,2, \ldots, 7)
\end{gathered}
$$

in each cell and the mass carried by each beam as

$$
\begin{gathered}
m_{1(i, j)}=a_{(i, j)} \rho_{i, j} \Delta A_{i, j}, \quad m_{\sigma, i, j}=b_{i, j} \rho_{i, j} \Delta A_{i, j} \\
(\sigma=2,3, \ldots, 7) .
\end{gathered}
$$

In the laboratory frame, the contribution of each $\sigma$ beam to the conservative state vector $Q$ in cell $(i, j)$ is

$$
Q_{\sigma, i, j}=\left(\begin{array}{c}
m_{\sigma, i, j} U_{\sigma 0, i, j} \\
m_{\sigma, i, j} U_{\sigma 0, i, j} U_{\sigma 1, i, j} \\
m_{\sigma, i, j} U_{\sigma 0, i, j} U_{\sigma 2, i, j} \\
m_{\sigma, i, j} U_{\sigma 0, i, j} U_{\sigma 3, i j} \\
m_{\sigma, i, j} U_{\sigma 0, i, j} U_{\sigma 0, i, j}
\end{array}\right),
$$

It is noted that $\sum_{\sigma=1}^{7} Q_{\sigma, i, j}=\Delta A_{i, j} Q_{i, j}$. During an interval of time $\Delta t$, these discrete beams will move and transfer
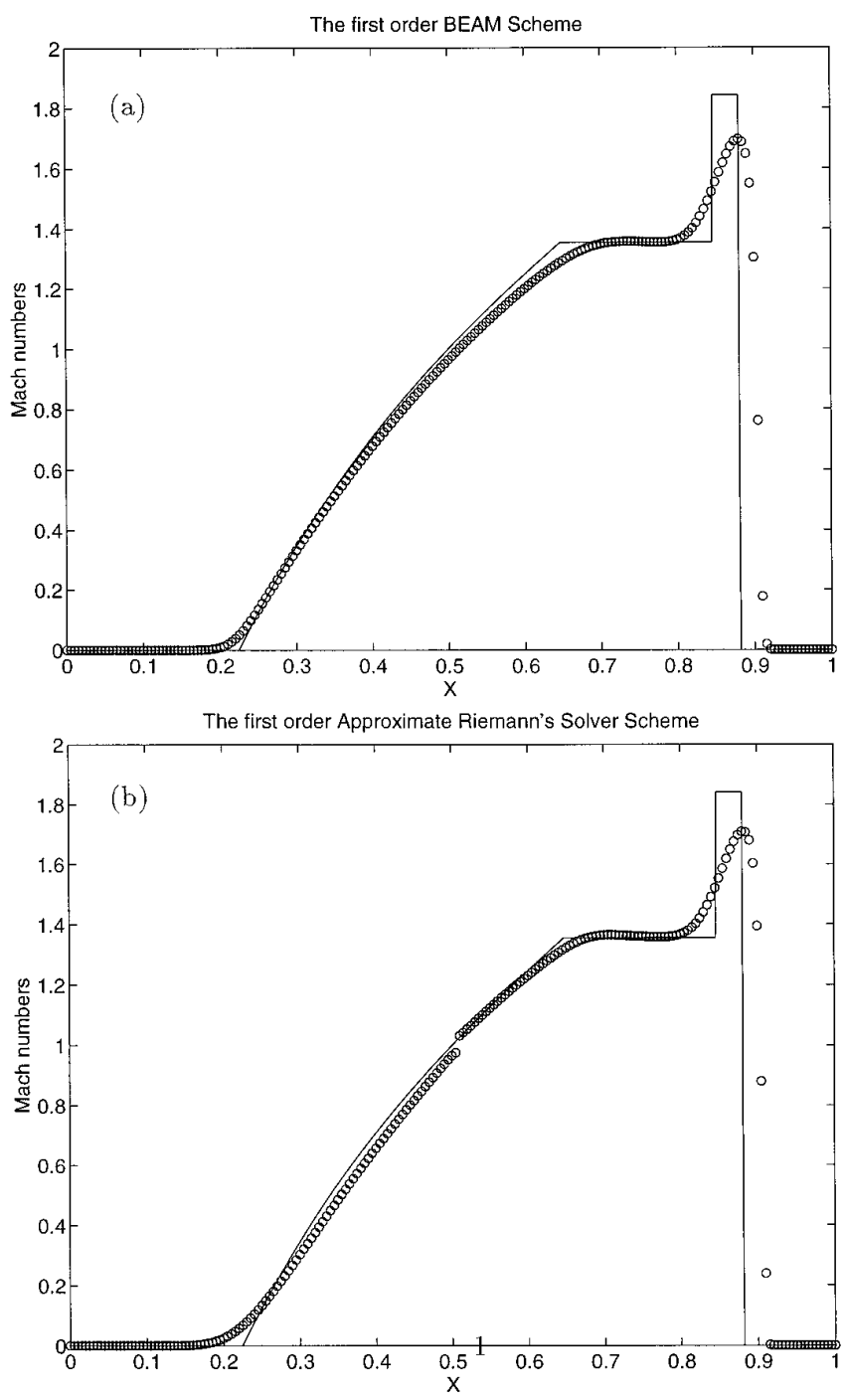

FIG. 3. Relativistic shock tube solution (Example 2). A comparison of first-order methods: (a) beam scheme; (b) Roe-type scheme without entropy fix.

mass, momentum, and energy into adjacent cells. The time step $\Delta t$ is subjected to the condition that no single beam moves farther than a cell size $\Delta A_{i, j}$ during $\Delta t$, i.e.,

$$
\Delta t \leq \min \left(\frac{\Delta x}{\left|V_{\sigma x}\right|}, \frac{\Delta y}{\left|V_{\sigma y}\right|}\right)
$$

which is the Courant-Friedrich-Lewy stability condition. During time step $\Delta t$ beam $\sigma(\sigma=1,2, \ldots, 7)$ in cell $(i, j)$ moves a distance either into cell $(i-1, j),(i+1, j),(i$, $j-1)$, or cell $(i, j+1)$ or remains in cell $(i, j)$ depending on the sign of the beam velocities. Define the fraction that transfers from cell $(i, j)$ to cells $(k=i \pm 1, j)$ as 
Density
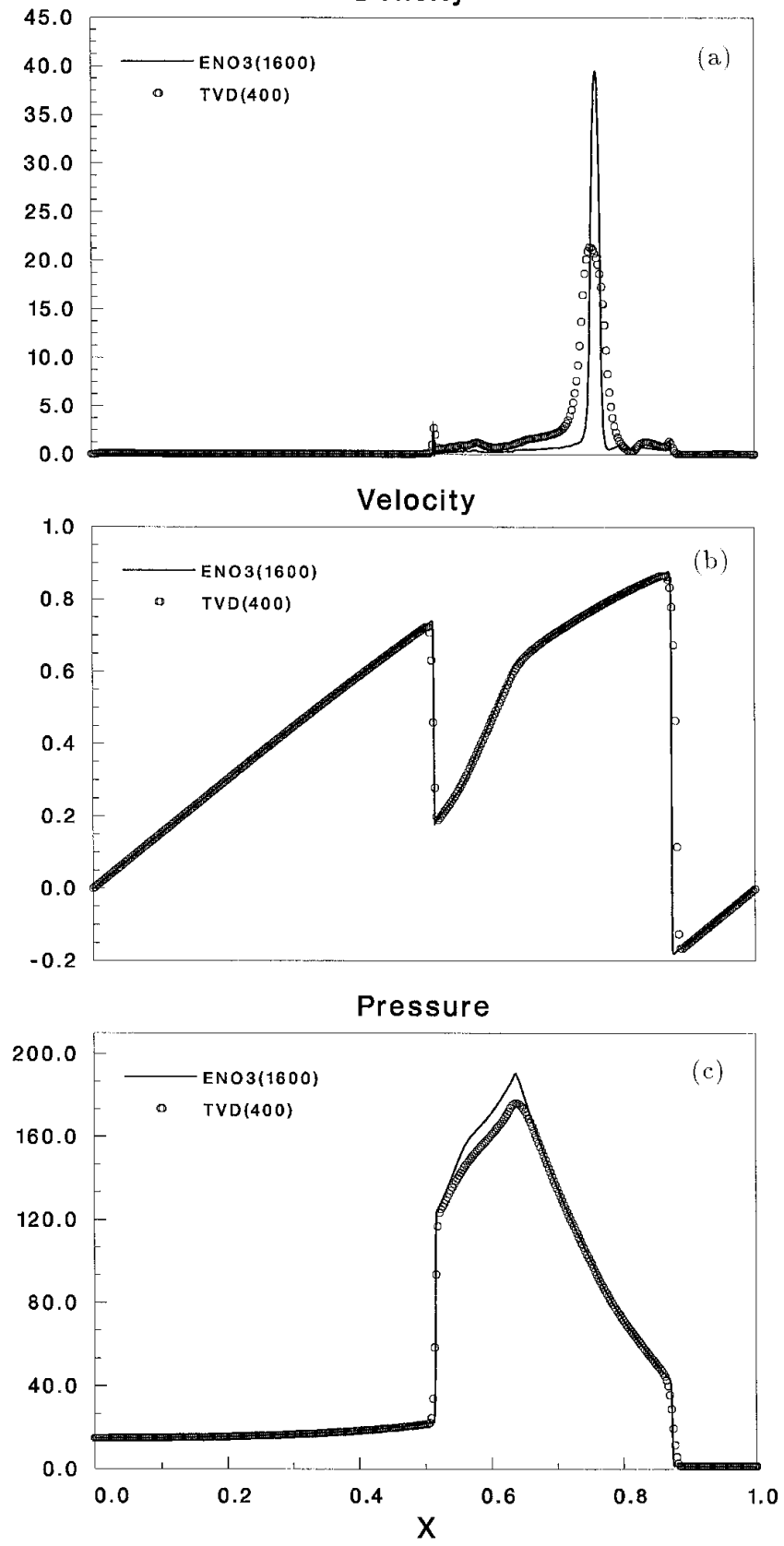

FIG. 4. Two interacting relativistic blast waves (Example 3) using TVD2; 400 points, $t=0.85$ (solid lines are the numerical solution of ENO3, 1600 points): (a) density; (b) velocity; (c) pressure.

$$
\alpha_{\sigma, i, j}^{i \pm 1, j}= \pm \frac{1}{2}\left(V_{\sigma x, i, j} \pm\left|V_{\sigma x, i, j}\right|\right) \frac{\Delta t}{\Delta x}= \pm V_{\sigma x, i, j}^{ \pm} \frac{\Delta t}{\Delta x}
$$

the fraction from cell $(i, j)$ to cells $(i, j \pm 1)$ as

$$
\alpha_{\sigma, i, j}^{i, j \pm 1}= \pm \frac{1}{2}\left(V_{\sigma y, i, j} \pm\left|V_{\sigma y, i, j}\right|\right) \frac{\Delta t}{\Delta y}=V_{\sigma y, i, j}^{ \pm} \frac{\Delta t}{\Delta y}
$$

Density
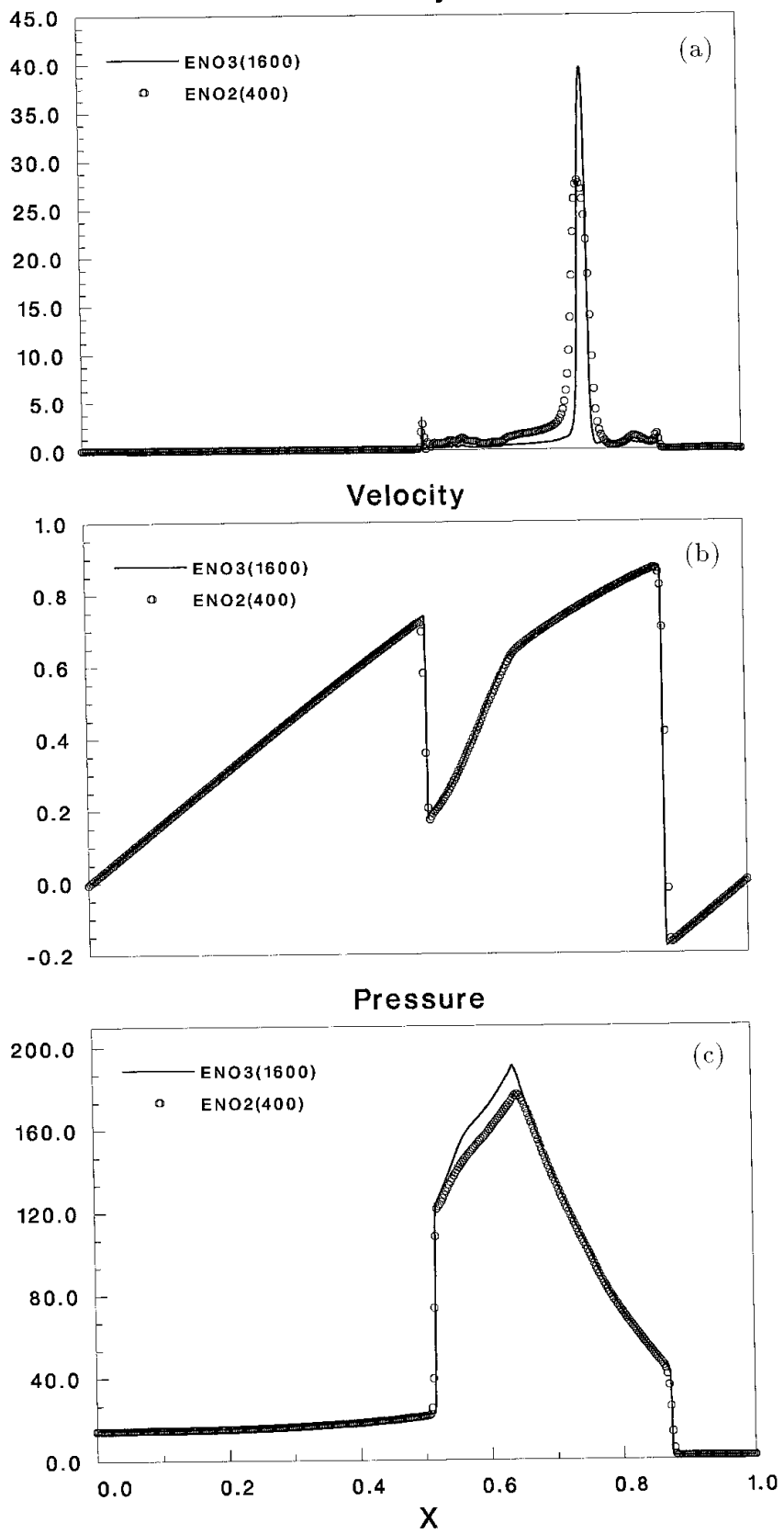

FIG. 5. Two interacting relativistic blast waves (Example 3) using ENO2; 400 points, $t=0.85$ (solid lines are the numerical solution of ENO3, 1600 points): (a) density; (b) velocity; (c) pressure.

and the fraction that remains in cell $(i, j)$ as

$$
\alpha_{\sigma, i, j}=1-\left(\left|V_{\sigma x, i, j}\right| \frac{\Delta t}{\Delta x}+\left|V_{\sigma y, i, j}\right| \frac{\Delta t}{\Delta y}\right) .
$$

After time step $\Delta t$, the new values of mass, momentum, 
and energy in each cell, $\left(Q_{i, j}^{n+1}\right)$, taking into account transfer of these quantities from adjacent cells are given by

$Q_{i, j}^{n+1}=\frac{1}{\Delta A_{i, j}} \sum_{\sigma=1}^{7}\left[\alpha_{\sigma, i, j}^{i, j} Q_{\sigma, i, j}^{n}+\sum_{\substack{k=i-1 \\ k \neq i}}^{i+1} \alpha_{\sigma, k, j}^{i, j} Q_{\sigma, k, j}^{n}+\sum_{\substack{l=j-1 \\ l \neq j}}^{j+1} \alpha_{\sigma, i, l}^{i, j} Q_{\sigma, i, l}^{n}\right]$,
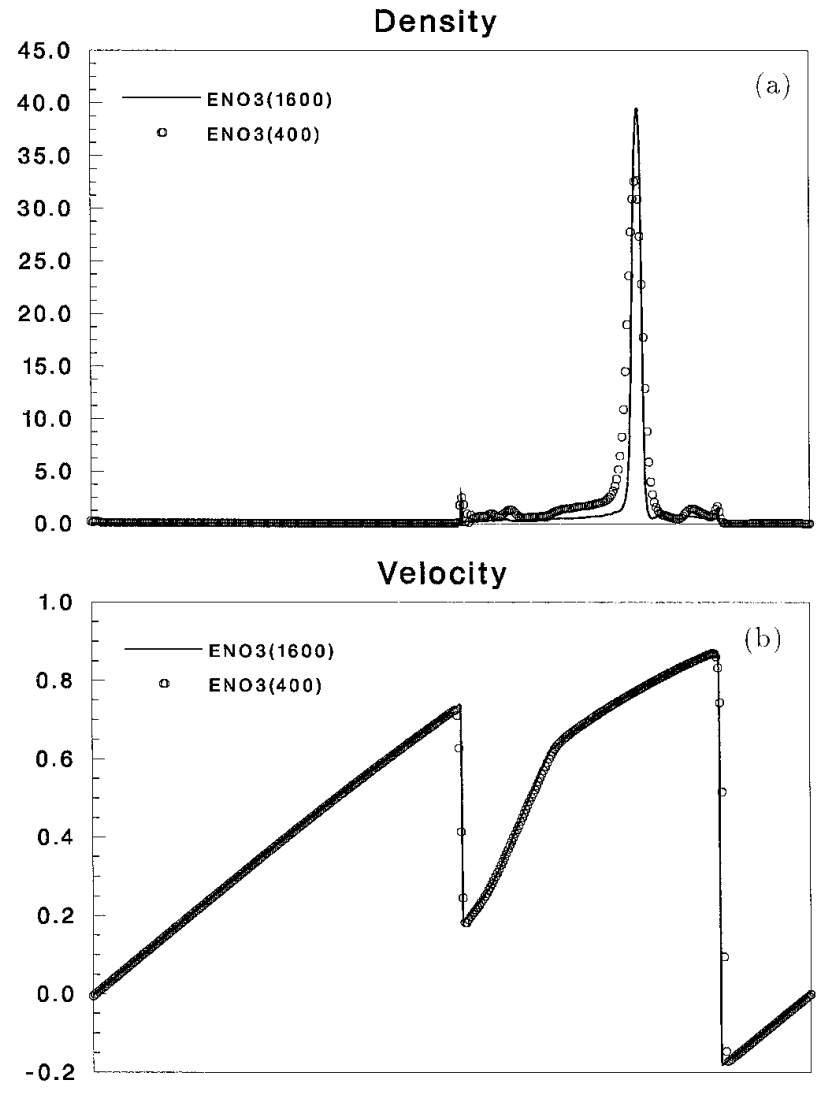

Pressure

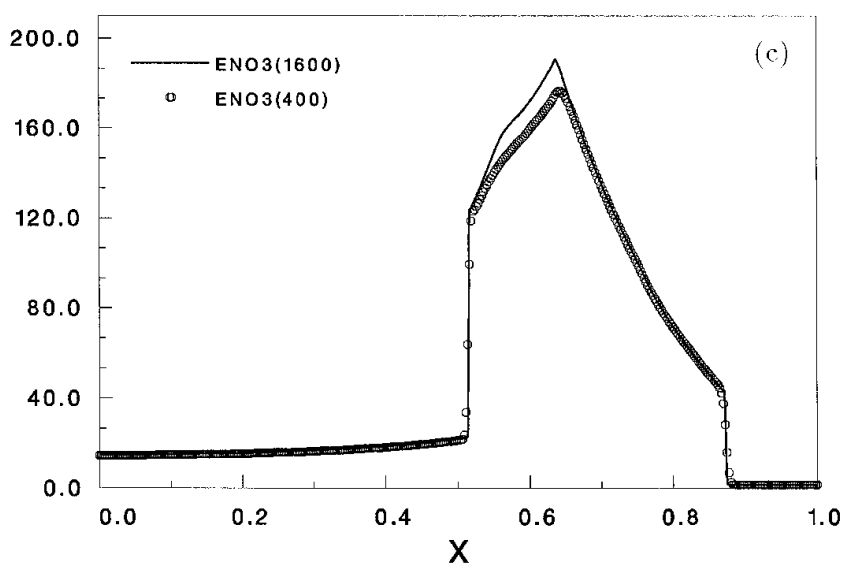

FIG. 6. Same as in Fig. 5 using ENO3.

\section{Lorentz factor}

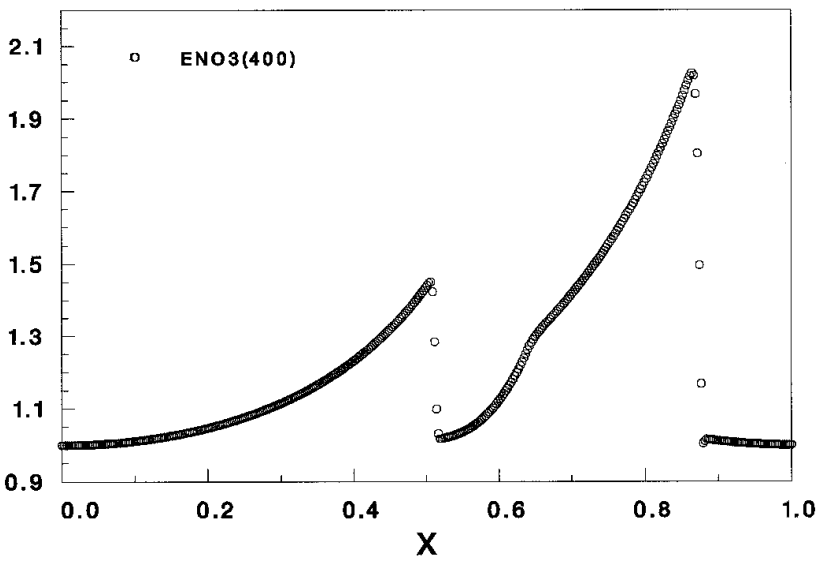

FIG. 7. Distribution of Lorentz factor in two interacting relativistic blast waves; ENO3, 400 points, $t=0.85$.

where

$$
Q_{i, j}^{n+1}=\left(\begin{array}{c}
\gamma_{u i, j} \rho_{i, j} \\
\gamma_{u i, j}^{2}\left(e_{i, j}+p_{i, j}\right) u_{x i, j} \\
\gamma_{u i, j}^{2}\left(e_{i, j}+p_{i, j}\right) u_{y i, j} \\
0 \\
\gamma_{u i, j}^{2}\left(e_{i, j}+p_{i, j}\right)-p_{i, j}
\end{array}\right)^{n+1}
$$

For problems in two space dimensions, the seven beams can be merged into five beams at this stage. Since $\mathbf{V}_{1, i, j}=$ $\mathbf{V}_{6, i, j}=\mathbf{V}_{7, i, j}$, we can combine these beams together and form a new single beam

$$
\tilde{Q}_{1, i, j}=Q_{1, i, j}+Q_{6, i, j}+Q_{7, i, j}
$$

The other four beams are kept unchanged

$$
\begin{array}{ll}
\tilde{Q}_{2, i, j}=Q_{2, i, j}, & \tilde{Q}_{3, i, j}=Q_{3, i, j}, \\
\tilde{Q}_{4, i, j}=Q_{4, i, j}, & \tilde{Q}_{5, i, j}=Q_{5, i, j} .
\end{array}
$$

Without causing any confusion we shall omit the superscript tilde in the equations to be described below and reset the number of the beams from seven to five, i.e., $\sigma=1,2,3,4,5$. Then we have

$$
Q_{\sigma=1}=\left(\begin{array}{c}
\rho \gamma_{u}\left(a+2 b \gamma_{\Delta}\right) \\
\rho \gamma_{u}^{2} u_{x}\left(a+2 b \gamma_{\Delta}^{2}\right) \\
\rho \gamma_{u}^{2} u_{y}\left(a+2 b \gamma_{\Delta}^{2}\right) \\
\rho \gamma_{u}^{2}\left(a+2 b \gamma_{\Delta}^{2}\right)
\end{array}\right) \text {, }
$$




$$
\begin{gathered}
Q_{\sigma=2}=\left(\begin{array}{c}
b \rho \gamma_{u} \gamma_{\Delta}\left(1+u_{x} \Delta v\right) \\
b \rho \gamma_{u} \gamma_{\Delta}\left(1+u_{x} \Delta v\right)\left[\gamma_{u} \gamma_{\Delta} u_{x}+\left(\gamma_{u} u_{x}^{2}+u_{y}^{2}\right) \gamma_{\Delta} \Delta v / u^{2}\right] \\
b \rho \gamma_{u} \gamma_{\Delta}\left(1+u_{x} \Delta v\right)\left[\gamma_{u} \gamma_{\Delta} u_{y}+\left(\gamma_{u}-1\right) u_{x} u_{y} \gamma_{\Delta} \Delta v / u^{2}\right] \\
b \rho \gamma_{u}^{2} \gamma_{\Delta}^{2}\left(1+u_{x} \Delta v\right)^{2}
\end{array}\right), \\
Q_{\sigma=3}=\left(\begin{array}{c}
b \rho \gamma_{u} \gamma_{\Delta}\left(1-u_{x} \Delta v\right) \\
b \rho \gamma_{u} \gamma_{\Delta}\left(1-u_{x} \Delta v\right)\left[\gamma_{u} \gamma_{\Delta} u_{x}-\left(\gamma_{u} u_{x}^{2}+u_{y}^{2}\right) \gamma_{\Delta} \Delta v / u^{2}\right] \\
b \rho \gamma_{u} \gamma_{\Delta}\left(1-u_{x} \Delta v\right)\left[\gamma_{u} \gamma_{\Delta} u_{y}-\left(\gamma_{u}-1\right) u_{x} u_{y} \gamma_{\Delta} \Delta v / u^{2}\right] \\
b \rho \gamma_{u}^{2} \gamma_{\Delta}^{2}\left(1-u_{x} \Delta v\right)^{2} \\
b \rho \gamma_{u} \gamma_{\Delta}\left(1+u_{y} \Delta v\right)
\end{array}\right), \\
Q_{\sigma=4}=\left(\begin{array}{c}
\left.\gamma_{y} \Delta v\right)\left[\gamma_{u} \gamma_{\Delta} u_{x}+\left(\gamma_{u}-1\right) u_{x} u_{y} \gamma_{\Delta} \Delta v / u^{2}\right] \\
b \rho \gamma_{u} \gamma_{\Delta}\left(1+u_{y}{ }^{2}\right. \\
b \rho \gamma_{u} \gamma_{\Delta}\left(1+u_{y} \Delta v\right)\left[\gamma_{u} \gamma_{\Delta} u_{y}+\left(u_{x}^{2}+\gamma_{u} u_{y}^{2}\right) \gamma_{\Delta} \Delta v / u^{2}\right] \\
b \rho \gamma_{u}^{2} \gamma_{\Delta}^{2}\left(1+u_{y} \Delta v\right)^{2} \\
b \rho \gamma_{u} \gamma_{\Delta}\left(1-u_{y} \Delta v\right)
\end{array}\right), \\
Q_{\sigma=5}=\left(\begin{array}{c}
\left.\gamma_{y} \Delta v\right)\left[\gamma_{u} \gamma_{\Delta} u_{x}-\left(\gamma_{u}-1\right) u_{x} u_{y} \gamma_{\Delta} \Delta v / u^{2}\right] \\
b \rho \gamma_{u} \gamma_{\Delta}\left(1-u_{y}\right. \\
b \rho \gamma_{u} \gamma_{\Delta}\left(1-u_{y} \Delta v\right)\left[\gamma_{u} \gamma_{\Delta} u_{y}-\left(u_{x}^{2}+\gamma_{u} u_{y}^{2}\right) \gamma_{\Delta} \Delta v / u^{2}\right] \\
b \rho \gamma_{u}^{2} \gamma_{\Delta}^{2}\left(1-u_{y} \Delta v\right)^{2}
\end{array}\right) .
\end{gathered}
$$

The beam velocities in the $x$-direction in the cell $(i, j)$ are

$$
\begin{gathered}
V_{1}^{x}=u_{x} \\
V_{2}^{x}=\frac{\gamma_{u} \gamma_{\Delta} u_{x}+\left(\gamma_{u} u_{x}^{2}+u_{y}^{2}\right) \gamma_{\Delta} \Delta v / u^{2}}{\gamma_{u} \gamma_{\Delta}\left(1+u_{x} \Delta v\right)} \\
\tilde{V}_{3}^{x}=\frac{\gamma_{u} \gamma_{\Delta} u_{x}-\left(\gamma_{u} u_{x}^{2}+u_{y}^{2}\right) \gamma_{\Delta} \Delta v / u^{2}}{\gamma_{u} \gamma_{\Delta}\left(1-u_{x} \Delta v\right)} \\
V_{4}^{x}=\frac{\gamma_{u} \gamma_{\Delta} u_{x}+\left(\gamma_{u}-1\right) u_{x} u_{y} \gamma_{\Delta} \Delta v / u^{2}}{\gamma_{u} \gamma_{\Delta}\left(1+u_{y} \Delta v\right)}, \\
\tilde{V}_{5}^{x}=\frac{\gamma_{u} \gamma_{\Delta} u_{x}-\left(\gamma_{u}-1\right) u_{x} u_{y} \gamma_{\Delta} \Delta v / u^{2}}{\gamma_{u} \gamma_{\Delta}\left(1-u_{y} \Delta v\right)},
\end{gathered}
$$

and the beam velocities in the $y$-direction in the cell $(i, j)$ are

$$
\begin{gathered}
V_{1}^{y}=u_{y}, \\
V_{2}^{y}=\frac{\gamma_{u} \gamma_{\Delta} u_{y}+\left(\gamma_{u}-1\right) u_{x} u_{y} \gamma_{\Delta} \Delta v / u^{2}}{\gamma_{u} \gamma_{\Delta}\left(1+u_{x} \Delta v\right)} \\
V_{3}^{y}=\frac{\gamma_{u} \gamma_{\Delta} u_{y}-\left(\gamma_{u}-1\right) u_{x} u_{y} \gamma_{\Delta} \Delta v / u^{2}}{\gamma_{u} \gamma_{\Delta}\left(1-u_{x} \Delta v\right)}, \\
V_{4}^{y}=\frac{\gamma_{u} \gamma_{\Delta} u_{y}+\left(u_{x}^{2}+\gamma_{u} u_{y}^{2}\right) \gamma_{\Delta} \Delta v / u^{2}}{\gamma_{u} \gamma_{\Delta}\left(1+u_{y} \Delta v\right)} \\
V_{5}^{y}=\frac{\gamma_{u} \gamma_{\Delta} u_{y}-\left(u_{x}^{2}+\gamma_{u} u_{y}^{2}\right) \gamma_{\Delta} \Delta v / u^{2}}{\gamma_{u} \gamma_{\Delta}\left(1-u_{y} \Delta v\right)} .
\end{gathered}
$$
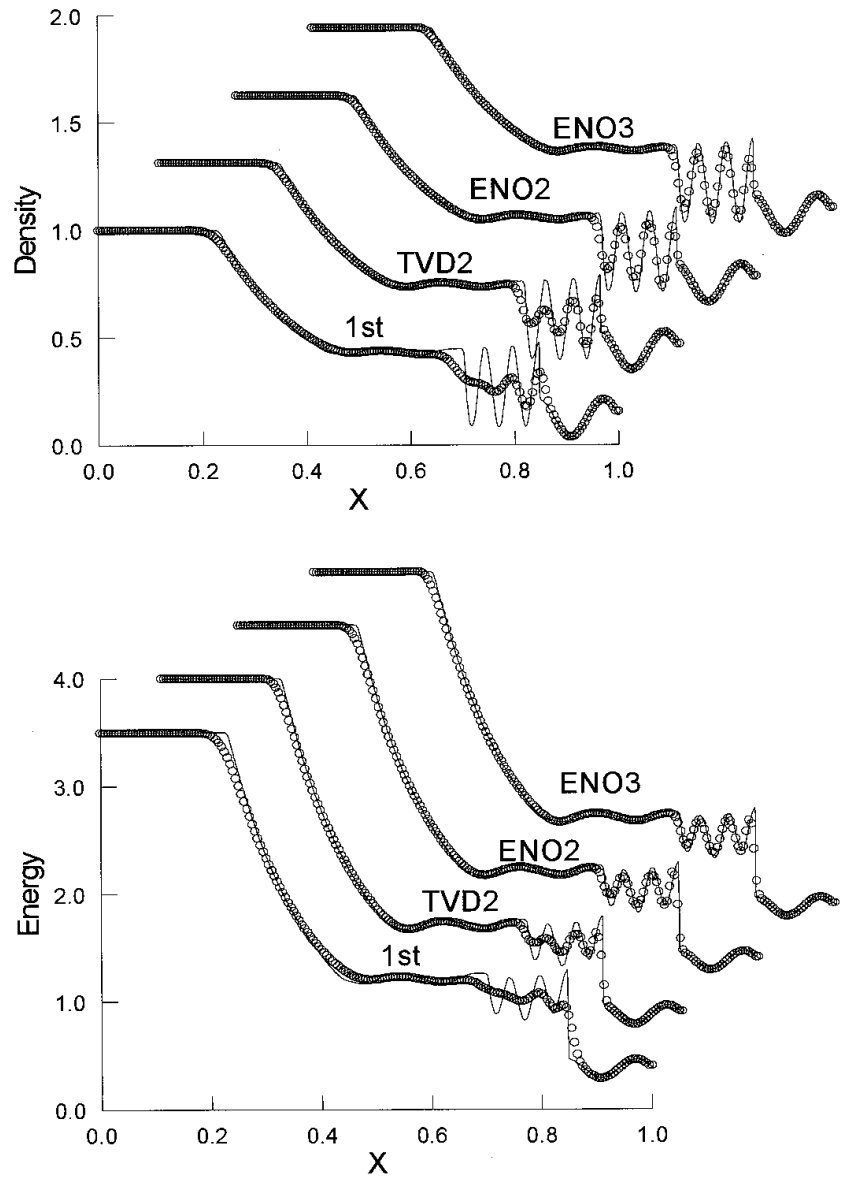

FIG. 8. Perturbed relativistic shock tube problem (Example 4) using beam schemes; 200 points.

The beam fluxes $F_{\sigma}$ and $G_{\sigma}$ in the $x$ - and $y$-direction are given by

$$
F_{\sigma}=V_{\sigma}^{x} Q_{\sigma}, \quad G_{\sigma}=V_{\sigma}^{y} Q_{\sigma}, \quad \sigma=1,2, \ldots, 5
$$

The integration scheme defined by Eq. (5.24) can also be cast into the form of a conservative scheme in terms of numerical fluxes

$$
\begin{aligned}
Q_{i, j}^{n+1}= & \frac{1}{\Delta A_{i, j}} \sum_{\sigma=1}^{5}\left[Q_{\sigma, i, j}^{n}-\frac{\Delta t}{\Delta x}\left(\tilde{F}_{\sigma, i+1 / 2, j}^{\mathrm{SP}}-\tilde{F}_{\sigma, i-1 / 2, j}^{\mathrm{SP}}\right)\right. \\
& \left.-\frac{\Delta t}{\Delta y}\left(\tilde{G}_{\sigma, i, j+1 / 2}^{\mathrm{SP}}-\tilde{G}_{\sigma, i, j-1 / 2}^{\mathrm{SP}}\right)\right],
\end{aligned}
$$

where the numerical flux in the $x$-direction, $\tilde{F}_{\sigma, i+1 / 2, j}^{\mathrm{SP}}$, is defined as 
$\mathbf{a}$
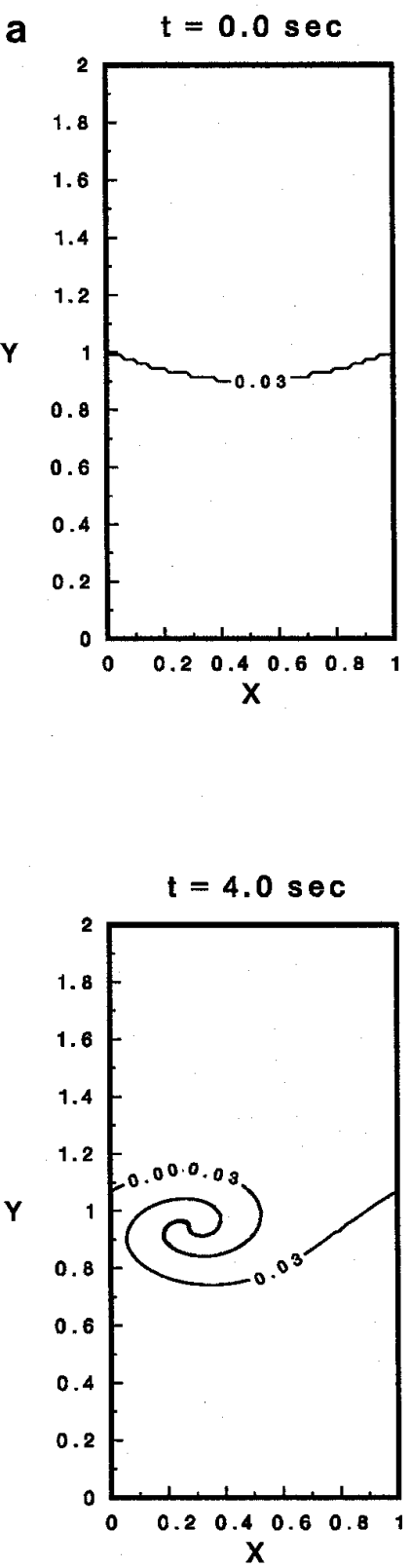

$t=1.0 \mathrm{sec}$

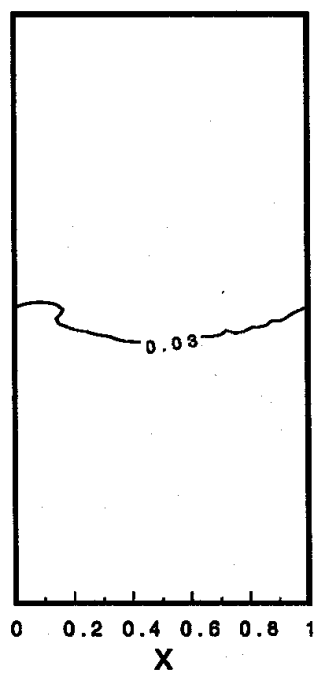

$t=5.0 \mathrm{sec}$

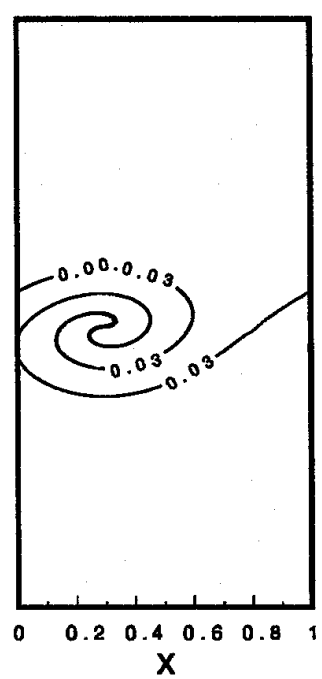

$t=2.0 \mathrm{sec}$

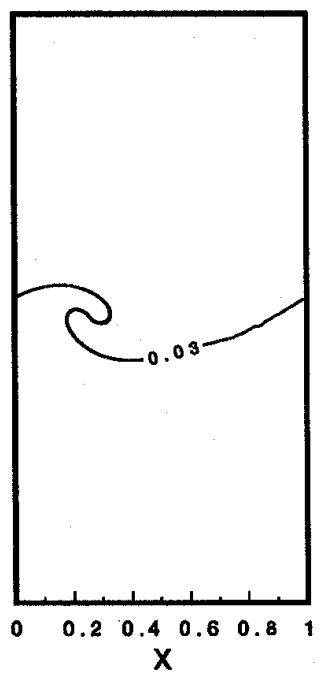

$t=6.0 \mathrm{sec}$

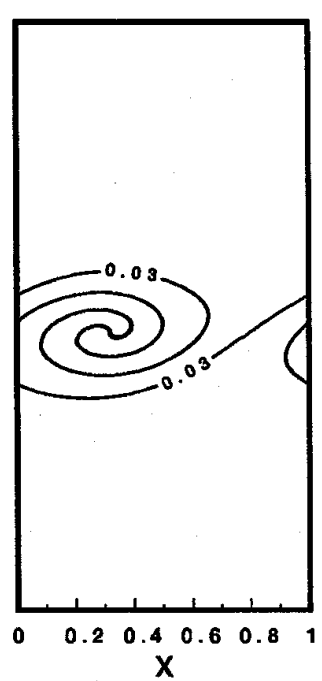

FIG. 9. Relativistic Kelvin-Helmholtz instability for air-air case on a $64 \times 128$ grid: (a) contours of the passive function $\phi$ at values $-\frac{1}{32}, 0, \frac{1}{32}$; (b) vorticity contours.

$$
\begin{aligned}
& \tilde{F}_{\sigma, i+1 / 2, j}^{\mathrm{SP}}=\frac{1}{2}\left(F_{\sigma, i, j}^{n}+F_{\sigma, i+1, j}^{n}-\Phi_{\sigma, i+1 / 2, j}^{\mathrm{SP}}\right), \\
& \Phi_{\sigma, i+1 / 2, j}^{\mathrm{SP}}=\left|V_{\sigma, i+1, j}^{x}\right| Q_{\sigma, i+1, j}-\left|V_{\sigma, i, j}^{x}\right| Q_{\sigma, i, j} .
\end{aligned}
$$

Similar expression can be defined for the numerical flux in the $y$-direction.

The scheme defined by Eq. (5.31) can be considered as a conservative upwind scheme for the following discrete set of conservation laws $\partial_{t} Q_{\sigma}+\partial_{x} F_{\sigma}\left(Q_{\sigma}\right)+\partial_{y} G_{\sigma}\left(Q_{\sigma}\right)=0, \quad \sigma=1,2, \ldots, 5$

where $Q_{\sigma}, F_{\sigma}$, and $G_{\sigma}$ are given above.

This completes the description of the first-order relativistic beam scheme in two space dimensions.

To advance the solution, the scheme defined by Eq. (5.31) is integrated using Strang-type dimensional splitting:

$$
Q_{\sigma, i, j}^{n+2}=L_{x}(\Delta t) L_{y}(\Delta t) L_{y}(\Delta t) L_{x}(\Delta t) Q_{\sigma, i, j}^{n}
$$



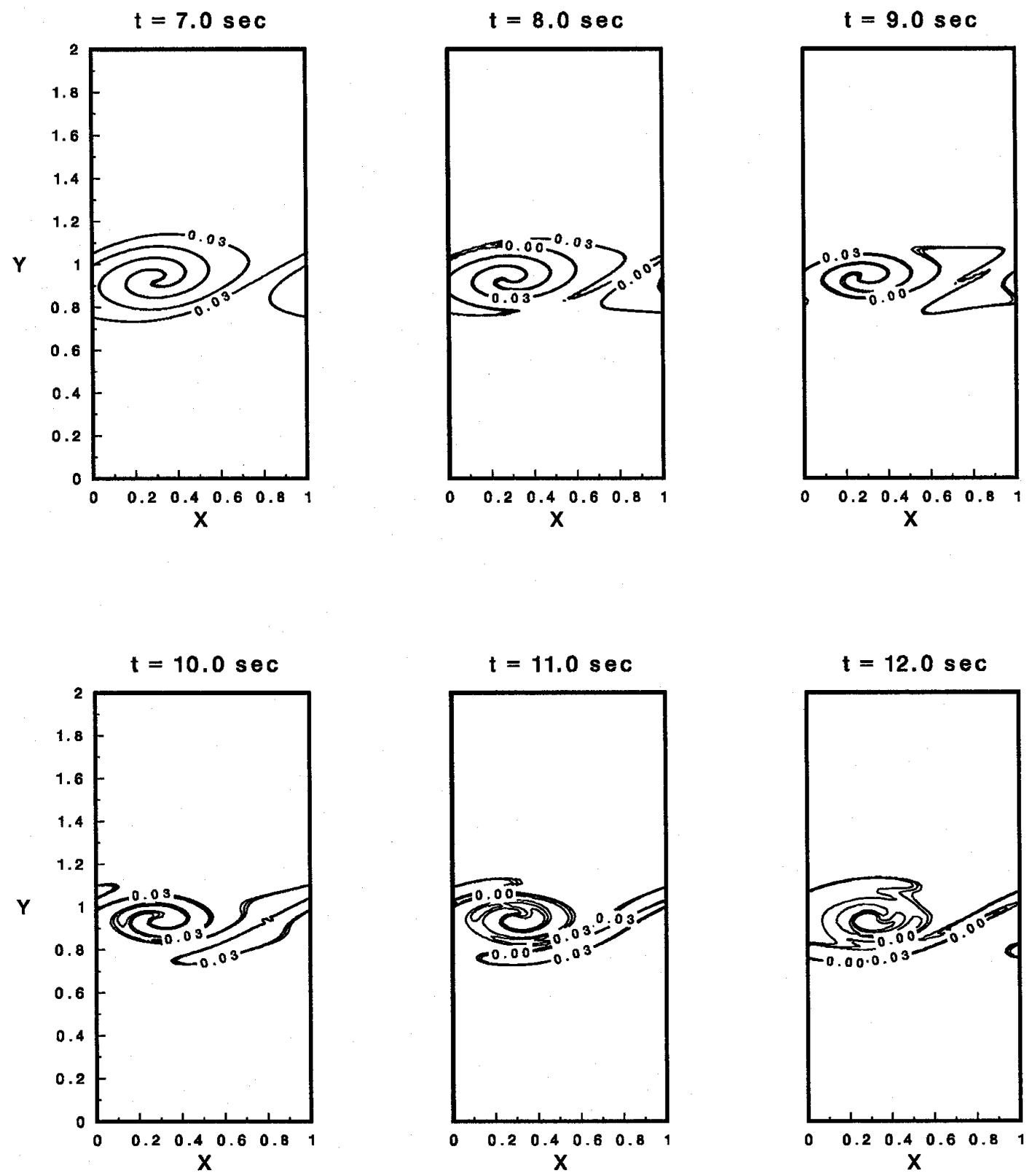

FIGURE 9-Continued

The one-dimensional operators $L_{x}$ and $L_{y}$ are defined by

$$
\begin{aligned}
Q_{\sigma, i, j}^{n+1} & =L_{x}(\Delta t) Q_{\sigma, i, j}^{n} \\
& =Q_{\sigma, i, j}^{n}-\frac{\Delta t}{\Delta x}\left(\tilde{F}_{\sigma, i+1 / 2, j}^{\mathrm{ENO}}-\tilde{F}_{\sigma, i-1 / 2, j}^{\mathrm{ENO}}\right), \\
Q_{\sigma, i, j}^{n+1} & =L_{y}(\Delta t) Q_{\sigma, i, j}^{n} \\
& =Q_{\sigma, i, j}^{n}-\frac{\Delta t}{\Delta y}\left(\tilde{G}_{\sigma, i, j+1 / 2}^{\mathrm{ENO}}-\tilde{G}_{\sigma, i, j-1 / 2}^{\mathrm{ENO}}\right) .
\end{aligned}
$$

After obtaining the new values of the macroscopic gas variables in the laboratory frame $Q_{i, j}^{n+1}=(R, M, N, E)_{i, j}$, for each cell, we need to calculate the local rest frame quantities $\rho, e, p, u_{x}$, and $u_{y}$ at time level $(n+1) \Delta t$. The laboratory quantities $R$ (mass density), $M, N$ (momentum density), and $E$ (energy density) are related to quantities in the local rest frame $e$ (energy density) and $\rho$ (particle density), and to the fluid velocity $\left(u_{x}, u_{y}\right)$ by the set of nonlinear transformations, Eqs. (5.5)-(5.8). The decoding procedure is similar to that described for one-dimensional case and will not be repeated here. 

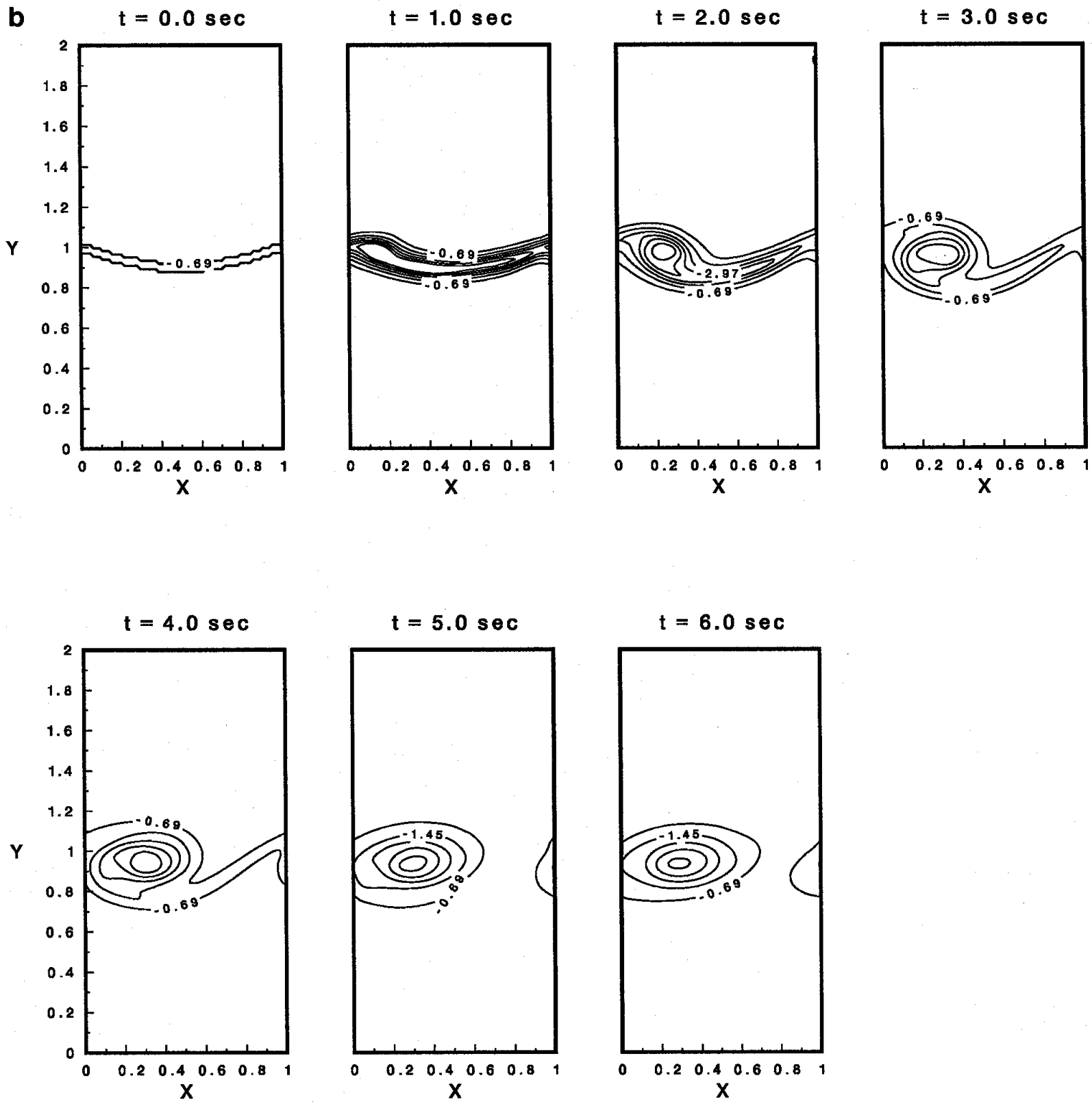

FIGURE 9-Continued

After the decoding and obtaining the local rest frame quantities, one then specifies that the local relativistic Maxwellian distribution with parameters determined by the new values of the gas variables and repeats the entire procedures to advance the solution in time.

\section{NUMERICAL EXPERIMENTS AND DISCUSSIONS}

In this section we report some numerical experiments to illustrate the performance of the present relativistic beam schemes. For validation and comparison we apply the nu- merical methods to relativistic Riemann problems in one space dimension which admit an analytical solution [1, $4,7,14]$. Relativistic flows in two space dimension are also included.

EXAMPLE 1 (Relativistic shock tube flow). We consider gas flows in a shock tube at relativistic velocities. In this problem a diaphragm, which is located at $x=0.5$, separates two regions, each in a constant equilibrium state at $t=0$. The initial conditions are specified as $\left(\rho_{L}, v_{L}, p_{L}, e_{L}\right)=$ $(1,0,1,3.5)$ for $0 \leq x \leq 0.5$ and $\left(\rho_{R}, v_{R}, p_{R}, e_{R}\right)=(0.125$, 

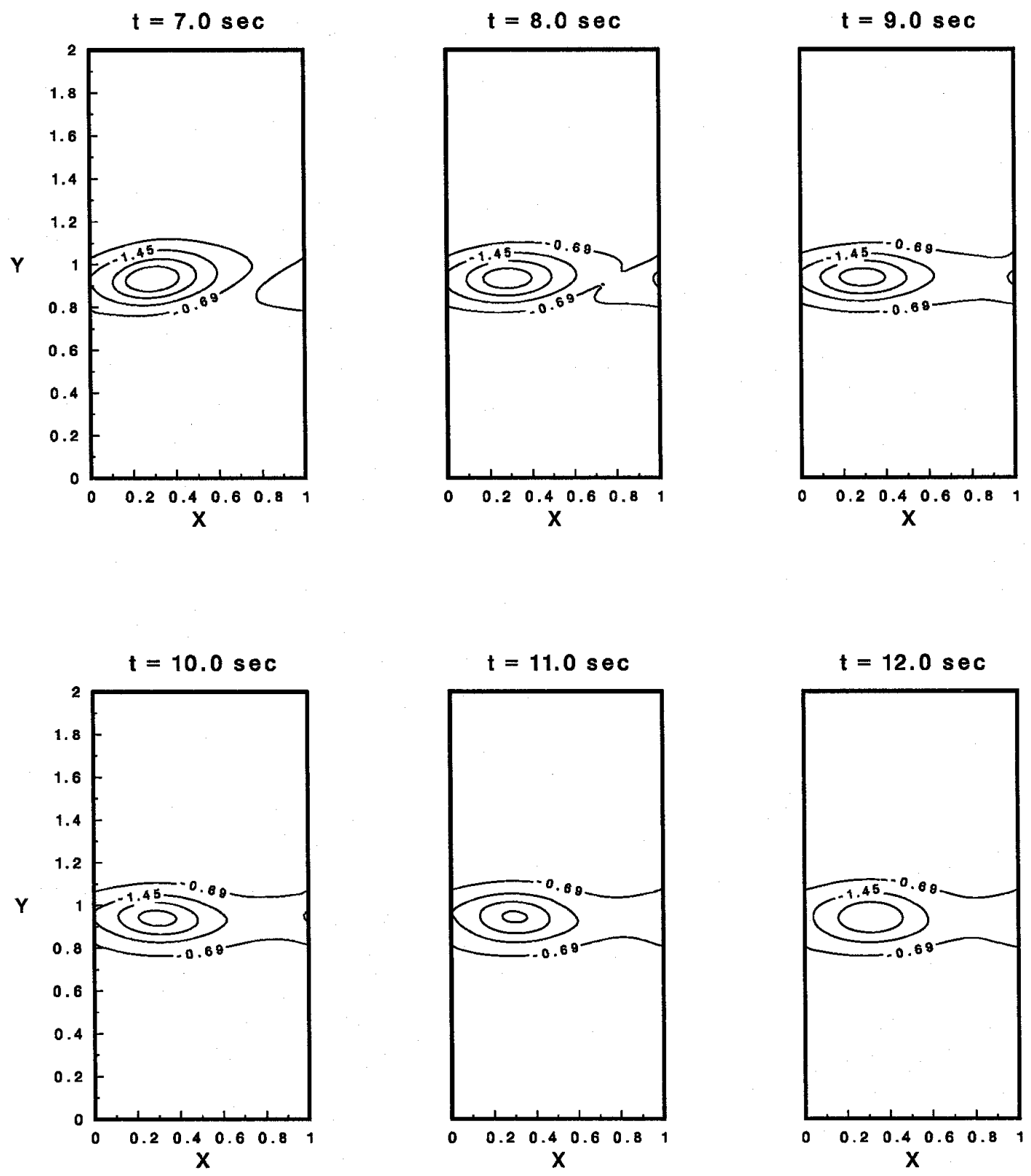

FIGURE 9-Continued

$0,0.1,0.375)$ for $0.5<x \leq 1$. This is a mildly relativistic case and the wave structures are quite similar to the nonrelativistic case, namely shock wave, contact surface, and rarefaction fan. The gamma law gas with $\Gamma=1.4$ was considered. We used 100 grid cells with $\Delta x=0.01$ and the CFL number was 0.8 . The results at time $t=0.48$ are ploted. The computed velocity, pressure, density, and energy profiles (circles) using the first-order upwind scheme (the original beam scheme), the second-order TVD2, the second-order ENO2, and the third-order ENO3 schemes are shown, respectively, in Fig. 1, together with the exact solutions (solid lines). The exact solution of the relativistic Riemann shock tube problems is obtained using the method described in [7]. The effect of high-order schemes TVD2, ENO2, and ENO3 can be seen through the more accurate representation of expansion fan and crisper shock and contact profiles.

EXAMPLE 2 (Relativistic shock tube flow with shock heating). This case includes shock heating of a cold fluid. The initial conditions are $\left(\rho_{L}, v_{L}, p_{L}, e_{L}\right)=(10,0,40 / 3,130 / 3)$ for $0 \leq x \leq 0.5$ and $\left(\rho_{R}, v_{R}, p_{R}, e_{R}\right)=\left(1 ., 0,2 / 3 \times 10^{-6}\right.$, 

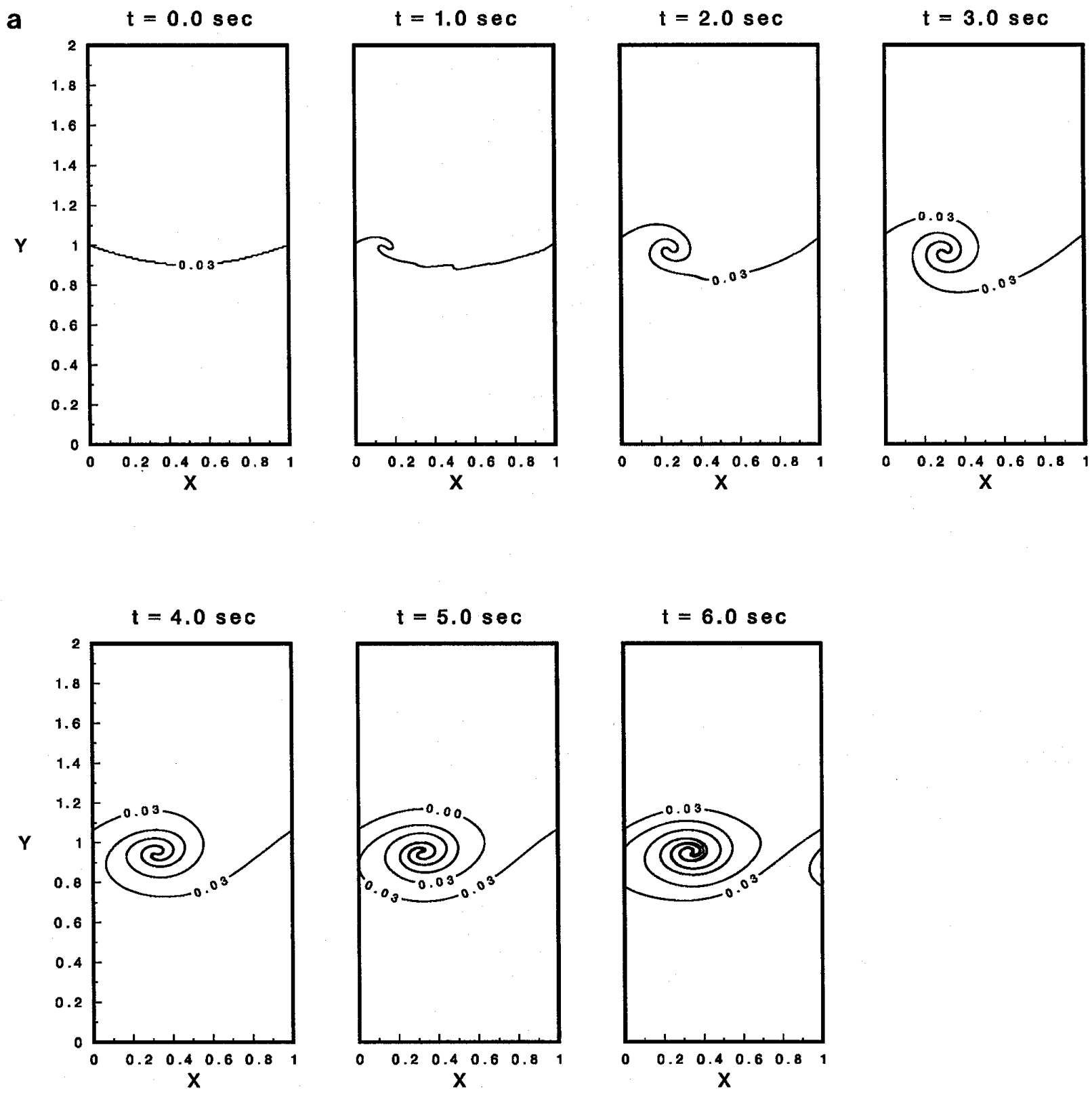

FIG. 10. Relativistic Kelvin-Helmholtz instability for air-air case on a $128 \times 256$ grid. (a) contours of passive function $\phi$ at values $-\frac{1}{32}$, 0 , $\frac{1}{32}$; (b) vorticity contours.

1) for $0.5 \leq x \leq 1$. The fluid velocity now slightly exceeds 0.7 and the relativistic effects are more pronounced than the previous case. We used 100 grid points with $\Delta x=0.01$ and $\Gamma=1.4$. The results of velocity, pressure, density, and energy profiles at time $t=0.48$ are shown in Fig. 2 for the first-order, second-order TVD2, second-order ENO2, and third-order ENO3 beam schemes, respectively. Again, the ability of the present methods to capture discontinuities is well confirmed and the effect of high-order methods is clearly demonstrated. This example contains an expansion fan which reaches supersonic velocities and a sonic transition occurs. The computed Mach number profiles obtained by using the first-order beam scheme and the Roe-type approximate relativistic Riemann solver [4] with 200 grid points are shown in Fig. 3. We observe that the Roe-type scheme gives an expansion shock when no entropy fix is applied.

EXAMPLE 3 (Two interacting relativistic blast waves). We consider here the interaction of two relativistic blast 

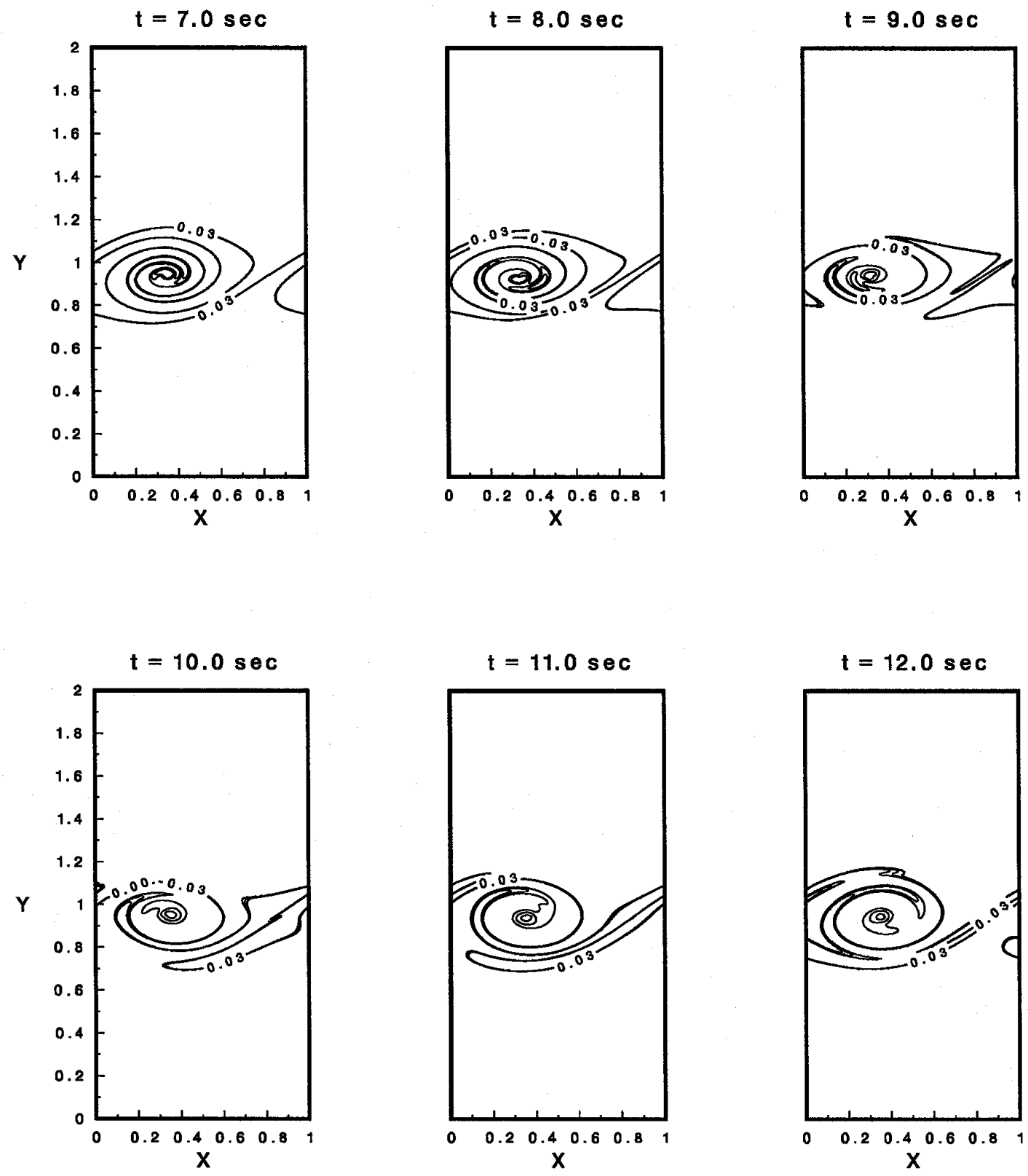

FIGURE 10-Continued

waves in which we follow the nonrelativistic case as first considered by Woodward and Collela [19]. The initial conditions are $\left(\rho_{L}, v_{L}, p_{L}\right)=\left(1 ., 0 ., 10^{3}\right)$ for $0<x<$ $0.1,\left(\rho_{M}, v_{M}, p_{M}\right)=\left(1 ., 0 ., 10^{-2}\right)$ for $0.1<x<0.9$, and $\left(\rho_{R}, v_{R}, p_{R}\right)=\left(1 ., 0 ., 10^{2}\right)$ for $0.9<x<1$. The reflective boundary condition is applied at both $x=0$ and $x=$ 1.0. See [19] for a detailed discussion of this problem in a nonrelativistic gas. Here, in the relativistic flow, the flow structures are quite different. We run this case with 400 grid points and $\mathrm{CFL}=0.9$ for a gamma-law gas
$\Gamma=1.4$. The results at the final time $t=0.85$ are shown in Figs. 4 to 6 for the TVD2, ENO2, and ENO3 schemes, respectively. The symbols (circles) are the computed data and the solid lines are the numerical solutions obtained using ENO3 with 1600 points. Notice that all three schemes have essentially converged for the velocity, but not for the pressure and the density. The density profiles indicate the severe smearing of contact discontinuities. However, the quality of resolution of the schemes are in the order of ENO3, ENO2, and TVD2. A plot of 
b
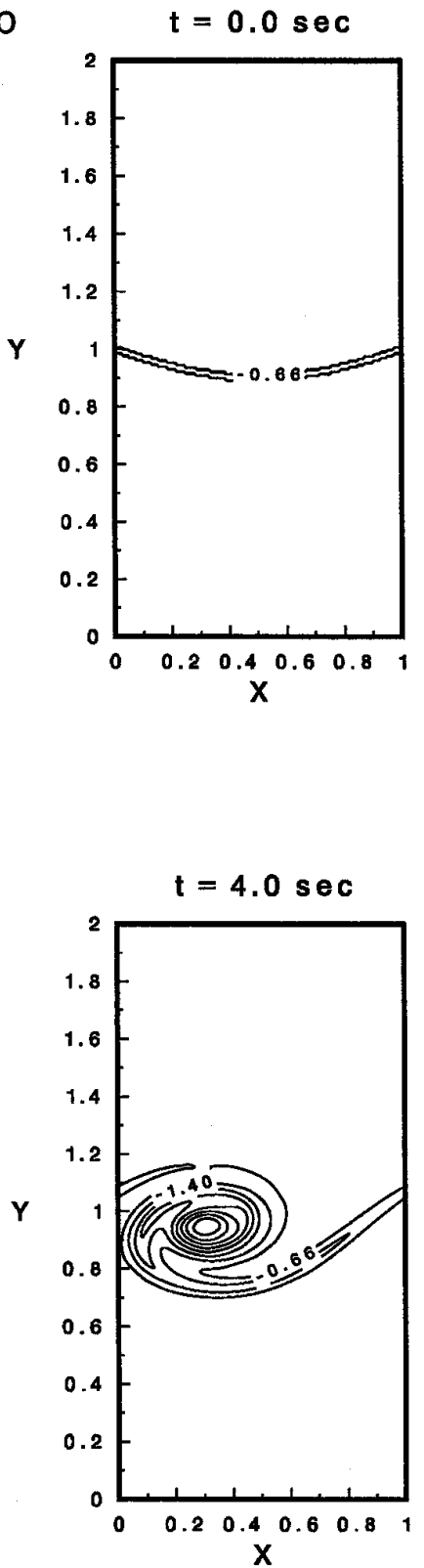

$\mathrm{t}=1.0 \mathrm{sec}$

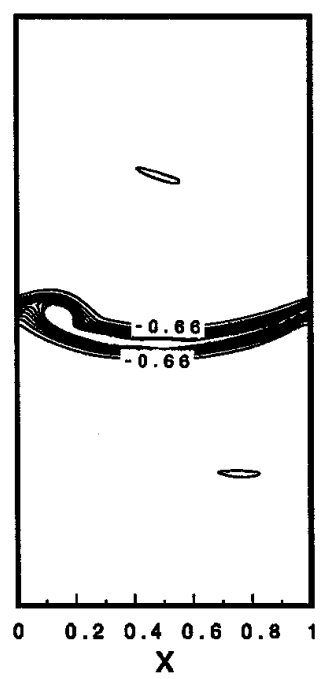

$t=5.0 \mathrm{sec}$

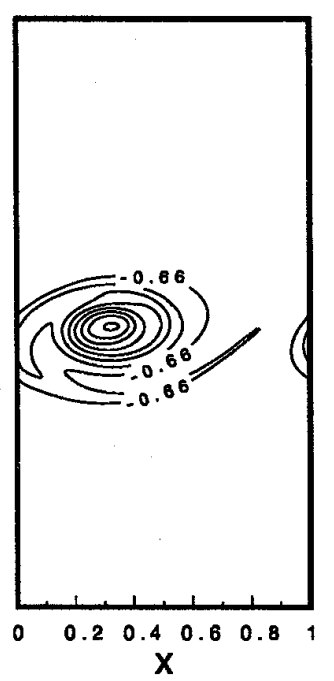

$t=2.0 \mathrm{sec}$

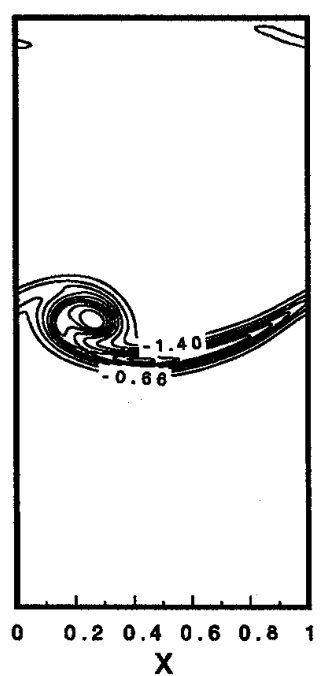

$t=6.0 \mathrm{sec}$

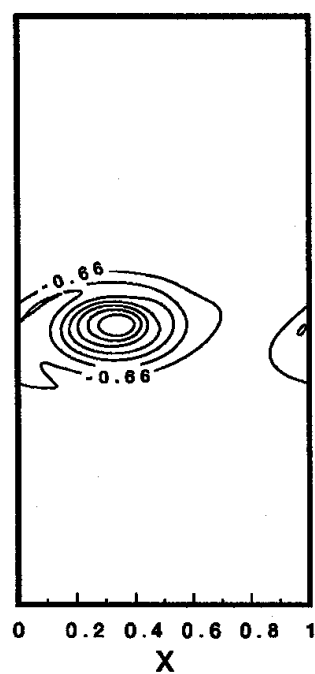

$t=3.0 \mathrm{sec}$

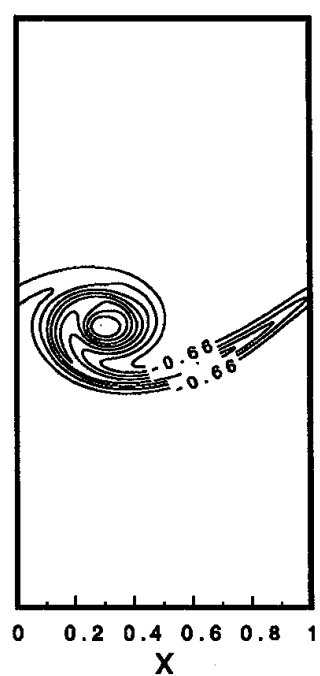

FIGURE 10-Continued

the Lorentz factor using the ENO3 with 400 points is shown in Fig. 7.

EXAMPLE 4 (Perturbed relativistic shock tube flow). This example is considered by Shu and Osher [15]. The initial conditions are $\left(\rho_{L}, v_{L}, p_{L}, e_{L}\right)=(1.0,0 ., 1.0,3.5)$ for $0 \leq x \leq 0.5$ and $\left(\rho_{R}, v_{R}, p_{R}, e_{R}\right)=\left(\rho_{R}, 0.0,0.1, e_{R}\right)$ for $0.5<x \leq 1$. Here the right state is a perturbed density field of sinusoidal wave, $\rho_{R}=0.125-0.0875 \sin (50(x-$ $0.5))$, and $e_{R}=p_{R} /(\Gamma-1)+\rho_{R}$. We run this test on meshes with 200 points and $\mathrm{CFL}=0.8$ for $\Gamma=1.4$. The computed solutions are plotted at time $t=0.48$. The results using TVD2, ENO2, and ENO3 are shown in Fig. 8. The solid lines, which are considered as exact solutions, are obtained using the ENO3 scheme with 2000 points. Slightly better resolution capability of the ENO3, as compared with that of ENO2, can be detected in the region where the shock wave is passing through and interacting with the fluctuating density field.

EXAMPLE 5 (Relativistic Kelvin-Helmholtz instability). Next, we consider problems in two space dimensions and 

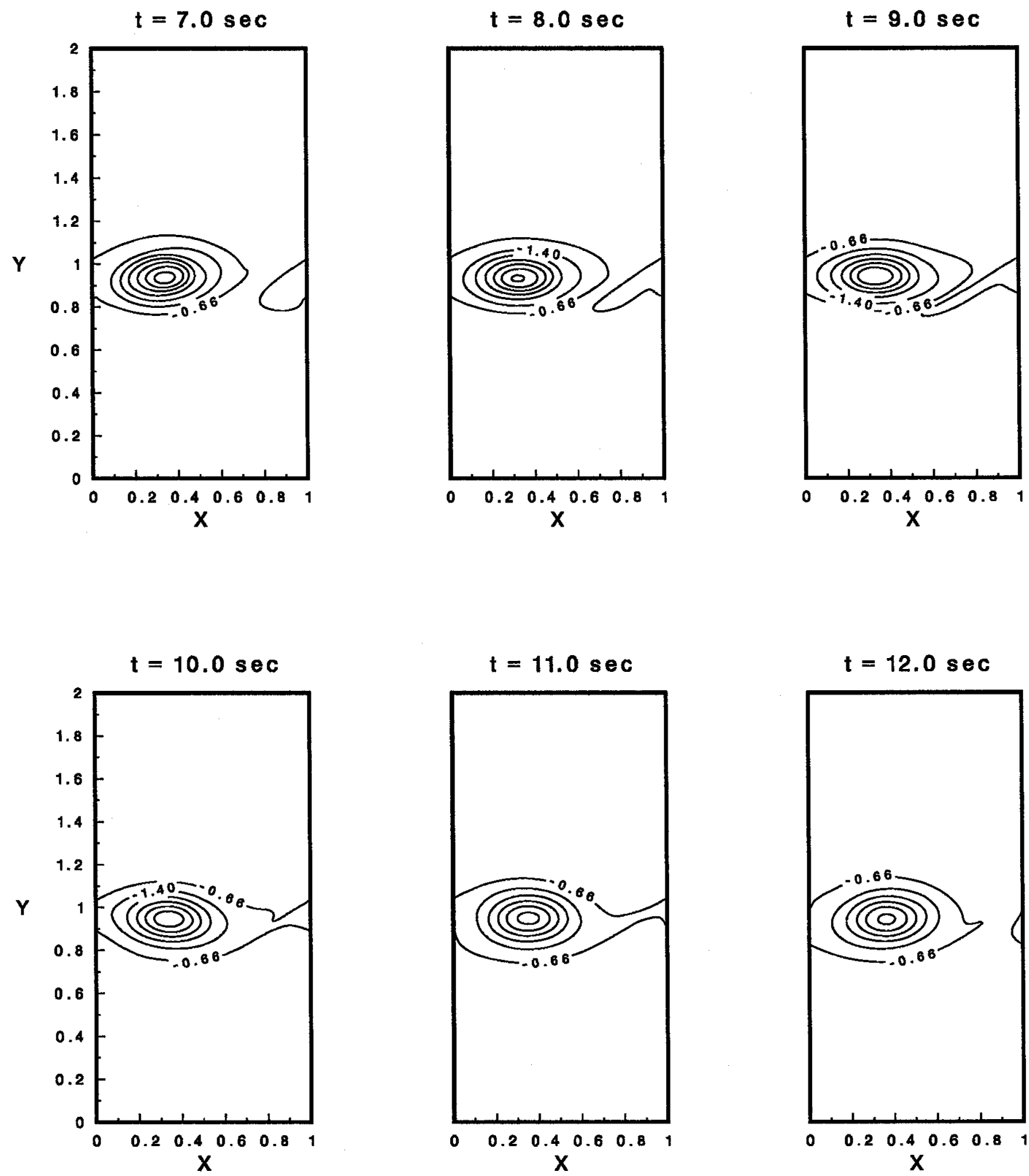

FIGURE 10-Continued

perform two-dimensional calculations of the relativistic Kelvin-Helmholtz instability flows. For this problem we follow the work of Mulder, Osher, and Sethian [10] for computing the propagating interface in nonrelativistic gas dynamics. The Kelvin-Helmholtz instability occurs when one fluid is moving at a different speed relative to another. Consider one fluid atop another; in between there is an interface and fluids are moving at different speeds initially parallel to the horizontal direction. As time goes on, the initial interface will roll up into a vortical structure, which serves to entrap the fluid. The initial conditions above and below the interface are $\left(\rho_{T}, u_{x T}, u_{y T}, p_{T}\right)=\left(1 ., u_{0}, 0 ., 1.0\right)$ and $\left(\rho_{B}, u_{x B}, u_{y B}, p_{B}\right)=\left(1 .,-u_{0}, 0 ., 1\right)$. Above the interface, the fluid moves towards the left with speed $u_{x T}=u_{0}$, and below the interface, the fluid moves towards the right with speed $u_{x B}=-u_{0}$. We assume an air-air case and set the density equal to 1 everywhere. We have periodic boundaries in the horizontal direction. The level set equation for a propagating interface is coupled to the set of conservation laws, Eq. (5.34), as

$$
\partial_{t}(R \phi)+\partial_{x}\left(R u_{x} \phi\right)+\partial_{y}\left(R u_{y} \phi\right)_{y}=0
$$


where the function $\phi(x, y, t)$ contains the embedded motion of propagating interface as the level set $\phi=0$. The evolution equation for $\phi$ is solved by using the same nonoscillatory scheme used to solve the relativistic Euler equations. We first used a $64 \times 128$ uniform grid. The initial shape of the interface is a sine perturbation with amplitude $A=$ 0.1 and $u_{0}=0.25 c$. The values of $\phi$ at $-\frac{1}{32}, 0, \frac{1}{32}$ are plotted in Fig. 9a. The results show the rollup of a vortex structure as it progresses several periods. The vorticity contours at different times are shown in Fig. 9b. The flow structures are qualitively similar to the nonrelativistic case [10]. A refine calculation using a fine mesh system of $128 \times 256$ grid points is also performed and the results are shown in Fig. 10. As compared with the base mesh calculations, the refinement in the flowfield structures can be detected.

\section{CONCLUDING REMARKS}

In this work, the first-order beam scheme of Sanders and Prendergast for nonrelativistic gas dynamics has been successfully extended to the relativistic gas dynamics. The resulting beam scheme provides an intrinsic entropy-satisfying mechanism and prevents the expansion shock from occurring. The beam scheme also splits the relativistic Euler equations into a discrete set of beam conservation laws. Formulations for two space dimensions are also included. A class of high-order relativistic beam schemes, including the total variation diminishing and essentially nonoscillatory methods, have also been devised. Numerical experiments with this class of high-order beam schemes for solving relativistic one-dimensional shock tube flows and twodimensional Kelvin-Helmholtz instability flows have been carried out and the high resolution of flow fields in all aspects have been demonstrated. The present relativistic beam scheme starts with a microscopic kinetic-theoretical description of particle motion and ends up with possessing the wave propagation property of macroscopic continuum description. Its particle-wave dual nature provides a new and interesting approach for treating relativistic gas dynamics.

\section{ACKNOWLEDGMENT}

This work was sponsored by the National Science Council of the Republic of China under Grant NSC 86-2612-E-002-010.

\section{REFERENCES}

1. D. S. Balsara, Riemann solver for relativistic hydrodynamics, J. Comput. Phys. 114, 284 (1994).

2. P. L. Bhatnagar, E. P. Gross, and M. Krook, Phys. Rev. 94, 511 (1964).

3. S. R. deGroot, W. A. van Leewen, and Ch. G. van Weert, Relativistic Kinetic Theory: Principles and Applications (North-Holland, New York, 1980).

4. A. Dolezal and S. S. M. Wong, Relativistic hydrodynamics and essentially non-oscillatory shock capturing schemes, J. Comput. Phys. 120, 266 (1995).

5. A. Harten, High resolution schemes for hyperbolic conservation laws, J. Comput. Phys. 49, 357 (1983).

6. A. Harten, B. Engquist, S. Osher, and S. R. Chakravathy, Uniform high order accurate essentially non-oscillatory schemes III, J. Comput. Phys. 71, 231 (1987).

7. J. F. Hawley, L. L. Smarr, and J. R. Wilson, A numerical study of nonspherical black hole accretion. I. Equations and test problems. Astrophys. J. 277, 296 (1984).

8. F. Jüttner, Ann. Phys. Chem. 34, 856 (1911).

9. L. D. Landau and E. M. Lifshitz, Fluid Mechanics (Pergamon, Elmsford, NY, 1987).

10. W. Mulder, S. Osher, and J. A. Sethian, Computing interface motion in compressible gas dynamics, J. Comput. Phys. 100, 209 (1992).

11. K. H. Prendergast and K. Xu, J. Comput. Phys. 109, 53 (1993).

12. P. L. Roe, J. Comput. Phys. 43, 357 (1981).

13. R. H. Sanders and K. H. Prendergast, The possible relation of the 3-kiloparsec arm to explosions in the galactic nucleus, Astrophys. $J$. 188, 489 (1974).

14. V. Schneider, U. Katscher, D. H. Rischke, B. Waldhauser, J. A. Maruhn, and C.-D. Munz, New algorithms for ultra-relativistic numerical hydrodynamics, J. Comput. Phys. 105, 92 (1993).

15. C.-W. Shu and S. Osher, Efficient implementation of essentially nonoscillatory shock-capturing schemes, II, J. Comput. Phys. 83, 32 (1989).

16. J. L. Synge, The Relativistic Gas (North-Holland, Amsterdam, 1957).

17. A. H. Taub, Relativistic Rankine-Hugoniot equations, Phys. Rev. 74, 328 (1948).

18. A. H. Taub, Relativistic fluid mechanics, Annu. Rev. Fluid Mech. 10, 301 (1978).

19. P. Woodward and P. Collela, The numerical simulation of two-dimensional fluid flow with strong shock, J. Comput. Phys. 51, 115 (1984).

20. K. Xu and K. H. Prendergast, J. Comput. Phys. 114, 9 (1994).

21. K. Xu, L. Martinelli, and A. Jameson, J. Comput. Phys. 120, 48 (1995).

22. J. Y. Yang and C. A. Hsu, High-resolution, non-oscillatory schemes for unsteady compressible flows, AIAA J. 30, 658 (1992).

23. J. Y. Yang, J. C. Huang, and L. Tsuei, Numerical solutions of the nonlinear model Boltzmann equations, Proc. Roy. Soc. Lond. A 448, 55 (1995).

24. J. Y. Yang and J. C. Huang, Rarefied flow computations using nonlinear model Boltzmann equations, J. Comput. Phys. 120, 323 (1995). 